\title{
Long noncoding RNA BLACAT2 promotes bladder cancer-associated lymphangiogenesis and lymphatic metastasis
}

\author{
Wang He, Guangzheng Zhong, Ning Jiang, Bo Wang, Xinxiang Fan, Changhao Chen, Xu Chen, Jian Huang, and Tianxin Lin \\ Department of Urology and the Guangdong Provincial Key Laboratory of Malignant Tumor Epigenetics and Gene Regulation, Sun Yat-sen Memorial Hospital, State Key Laboratory of Oncology in \\ South China, Sun Yat-sen University, Guangzhou, Guangdong, China.
}

\begin{abstract}
The prognosis for bladder cancer patients with lymph node (LN) metastasis is dismal and only minimally improved by current treatment modalities. Elucidation of the molecular mechanisms that underlie LN metastasis may provide clinical therapeutic strategies for LN-metastatic bladder cancer. Here, we report that a long noncoding RNA LINC00958, which we have termed bladder cancer-associated transcript 2 (BLACAT2), was markedly upregulated in LN-metastatic bladder cancer and correlated with LN metastasis. Overexpression of BLACAT2 promoted bladder cancer-associated lymphangiogenesis and lymphatic metastasis in both cultured bladder cancer cell lines and mouse models. Furthermore, we demonstrate that BLACAT2 epigenetically upregulated VEGF-C expression by directly associating with WDR5, a core subunit of human H3K4 methyltransferase complexes. Importantly, administration of an anti-VECF-C antibody inhibited LN metastasis in BLACAT2overexpressing bladder cancer. Taken together, these findings uncover a molecular mechanism in the lymphatic metastasis of bladder cancer and indicate that BLACAT2 may represent a target for clinical intervention in LN-metastatic bladder cancer.
\end{abstract}

\section{Introduction}

Bladder cancer is one of the most common genitourinary malignancies, with approximately 429,800 new cases and 165,100 deaths annually worldwide (1). Bladder cancer is categorized into 2 groups based on its distinct behavior: low-grade nonmuscle-invasive bladder cancer (NMIBC) and high-grade muscle-invasive bladder cancer (MIBC). NMIBC is the most common presentation of bladder cancer and is often treatable by endoscopic resection and intravesical therapy. However, the probability of death from MIBC with LN metastasis is markedly higher than that from MIBC without LN metastasis. The death rate increases from $18.6 \%$ to $77.6 \%$ within 5 years, even when the MIBC is treated with radical cystectomy $(2,3)$, and LN-metastatic MIBC patients with extranodular extensions have an even worse prognosis (hazard ratio: 2.01) (4). Multiple independent studies have demonstrated that $\mathrm{LN}$ metastasis is a key prognostic factor in bladder cancer $(3,5,6)$.

LN metastasis is a complex multistep process that involves intratumoral and peritumoral lymphangiogenesis, dissemination of tumor cells to lymphatic vessels, transport of tumor cells to LNs through the lymphatic system, and settlement and colonization expansion of tumor cells in LNs $(7,8)$. It has been verified that lymphangiogenesis, which is correlated with LN metastasis and metastasis-free survival in bladder cancer, is a necessary step for LN metastasis of human cancer (9-11). Accumulating evidence

Authorship note: W. He, G. Zhong, and N. Jiang contributed equally to this study. Conflict of interest: The authors have declared that no conflict of interest exists. Submitted: July 19, 2017; Accepted: December 7, 2017.

Reference information: J Clin Invest. 2018;128(2):861-875.

https://doi.org/10.1172/JCI96218. has shown that upregulated VEGF-C, a lymphangiogenic growth factor, positively correlates with regional LN metastasis and poor survival in multiple human malignancies, including bladder cancer $(9,11-15)$. Several groups have reported that when VEGF-C signaling is blocked via either small interfering RNAs or a neutralizing antibody to VEGF-C or VEGF-R3, the lymphatic-based metastatic spread of human malignancies can be inhibited (9,16-18). Importantly, the VEGF-C monoclonal antibody (VGX-100) has been tested in a phase I clinical trial for advanced or metastatic solid tumors that are refractory to standard treatments (ClinicalTrials.govNCT01514123). Therefore, understandingtheregulatory mechanisms of VEGF-C in bladder cancer may provide clinically valuable predictive tools for effective anti-VEGF-C treatments.

Long noncoding RNAs (lncRNAs), defined as transcripts that are larger than $200 \mathrm{nt}$ with no protein-coding capacity, play diverse roles in the development and progression of human cancers (19). For example, HOTAIR, SChLAP1, and BLACAT1 participate in the metastatic cascade by promoting cell migration and invasion (20-22), and lncRNA-ATB and LINC01186 contribute to metastasis by inducing epithelial-mesenchymal transition (EMT) $(23,24)$. However, the precise mechanisms of lncRNAs in lymphangiogenesis remain unknown.

Herein, we identified an lncRNA LINC00958, which we termed bladder cancer-associated transcript 2 (BLACAT2), which was markedly overexpressed in LN-metastatic bladder cancer. Overexpression of BLACAT2 promoted bladder cancer-associated lymphangiogenesis and lymphatic metastasis both in vitro and in vivo. Importantly, blocking VEGF-C signaling with a VEGF-C antibody dramatically reduced LN metastasis of high BLACAT2- expressing bladder cancers in vivo. Therefore, our results indicate that BLACAT2 plays a crucial role in lymphangiogenesis and lymphat- 
A

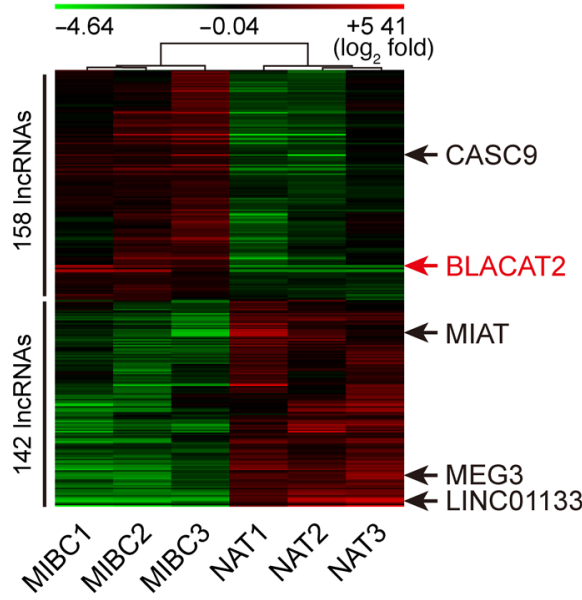

D

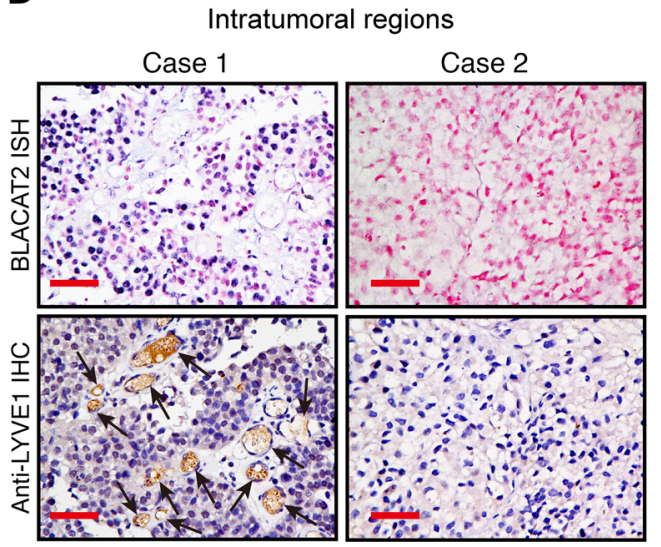

B

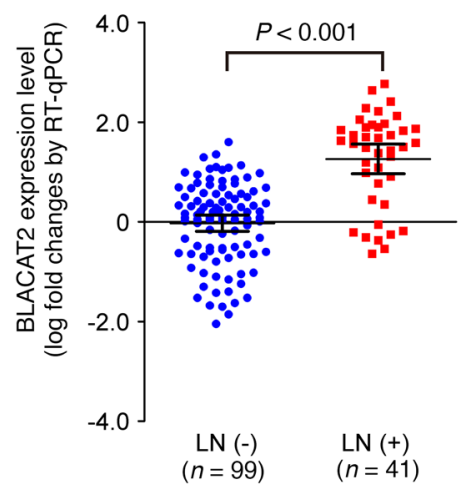

C

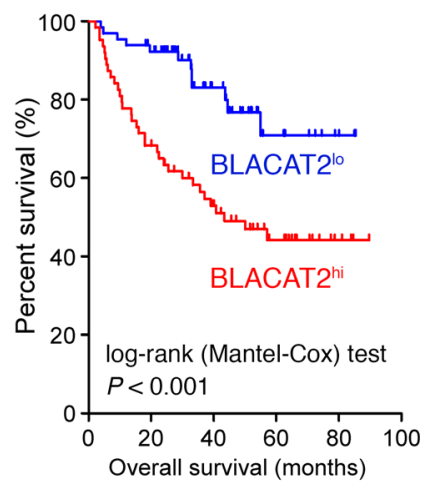

$\mathbf{E}$

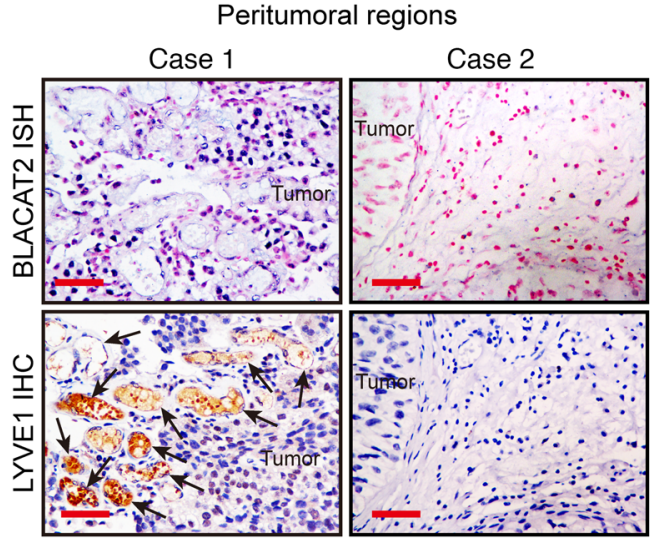

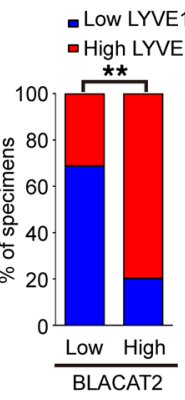

Figure 1. BLACAT2 overexpression correlates with LN metastasis and poor prognosis of bladder cancer. (A) Unsupervised hierarchical clustering of IncRNAs that are differentially expressed in MIBC and paired normal adjacent tissues (NAT) (fold changes $>2.0, P<0.05$ ). The red color scale (log, fold change) represents a higher expression level, and the green color scale represents a lower expression level. (B) RT-qPCR analysis of BLACAT2 expression in a 140-case cohort of freshly collected human bladder cancer samples with or without LN metastasis. The nonparametric Mann-Whitney $U$ test was used to compare the expression levels of the 2 groups. (C) Negative correlation between BLACAT2 expression and survival in the patient cohort referred to in B. The Kaplan-Meier method was used to estimate survival for the 2 groups. Median BLACAT2 expression was used as a cutoff value. (D and E) Representative images (left panels) and percentages (right panels) of tissue specimens with high and low levels of LYVE1-positive intratumoral (D) and peritumoral (E) microlymphatic vessels in 140 cases of bladder cancer with low or high expression of BLACAT2. BLACAT2 expression levels were quantified by ISH, and microlymphatic vessel density was quantified by immunohistochemistry using the anti-LYVE1 antibody. Two representative cases are shown. Statistical significance was assessed by $\chi^{2}$ test. Scale bars: $50 \mu \mathrm{m}$ (D and $\left.\mathbf{E}\right) .{ }^{* *} P<0.01$.

ic metastasis, and our data highlight a regulatory mechanism for VEGF-C and LN metastasis in bladder cancer.

\section{Results}

BLACAT2 overexpression correlates with LN metastasis of bladder cancer. To identify critical lncRNAs that contribute to bladder cancer progression, high-grade MIBC and paired adjacent normal tissues from 3 patients were subjected to an lncRNA microarray analysis. The patient characteristics are shown in Supplemental Table 1 (supplemental material available online with this article; https://doi.org/10.1172/JCI96218DS1). As shown in Figure 1A, the microarray analysis identified 158 upregulated and 142 downregulated lncRNAs from 7,419 analyzed lncRNAs in the MIBC tissues, including CASC9, MIAT, MEG3, and LINC01133, which are associated with human cancer progression (25-28). Additionally, one lncRNA (LINC00958, RefSeq accession number NR_038904), here termed BLACAT2, was significantly upregulated in highgrade MIBC tissues compared with paired adjacent normal tissues (Figure 1A). Furthermore, quantitative reverse-transcription PCR (RT-qPCR) analysis showed that BLACAT2, which was examined in a larger 140-case cohort of bladder cancers, was overexpressed in high-grade bladder cancer compared with lower-grade tumor, in LN-positive bladder cancer compared with LN-negative tumor, and in LN-metastatic tumors compared with matched primary tumors (Figure 1B and Supplemental Figure 1, A and B). This result was further confirmed by analyses of The Cancer Genome Atlas (TCGA) database (Supplemental Figure 1C). Moreover, statistical analysis revealed that BLACAT2 expression was not only strongly correlated with pathological grade $(P<0.001)$, but was also associated with the LN metastasis status of bladder cancer $(P$ $<$ 0.05) (Supplemental Table 2). Furthermore, patients with high BLACAT2-expressing bladder cancers had shorter overall and 
A

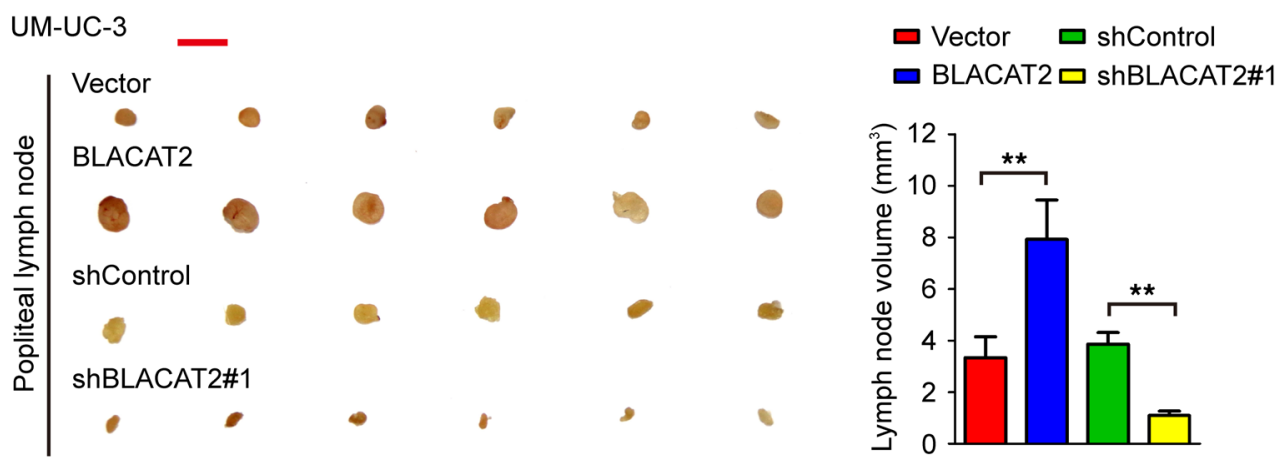

B

UM-UC-3
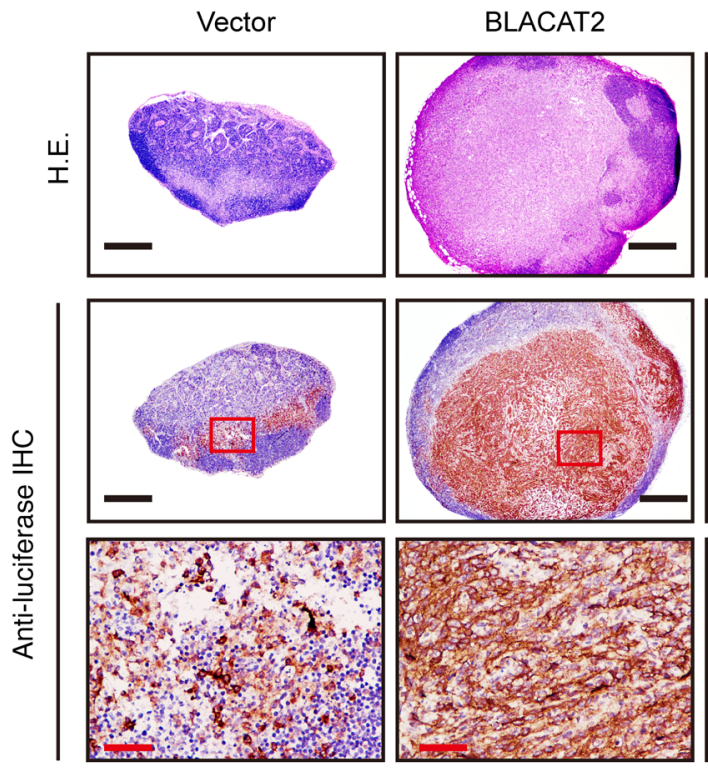

shControl
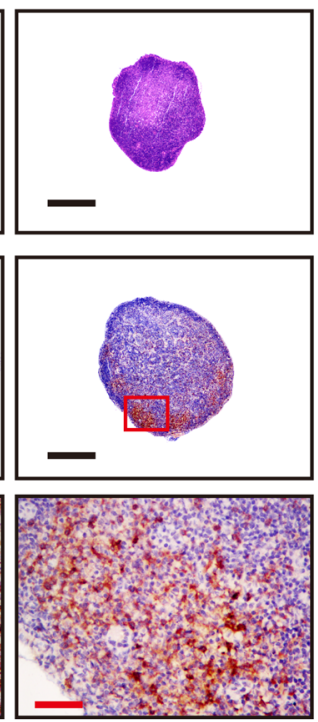

shBLACAT2\#1
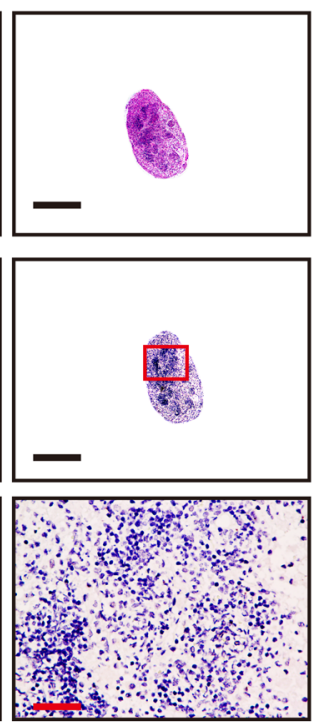

Figure 2. BLACAT2 overexpression promotes LN metastasis of bladder cancer cells in vivo. (A) Representative images of enucleated popliteal LNs (left panel) inoculated with the indicated cells ( $n=12$ per group) and histogram analysis of the $L N$ volume (right panel). Scale bar: $5 \mathrm{~mm}$. Error bars indicate SD of the mean. Statistical significance was assessed using 2-tailed Student's $t$ test. ${ }^{* *} P<0.01$.

(B) Representative images of the popliteal LNs analyzed by $\mathrm{H} \& \mathrm{E}$ staining and $\mathrm{IHC}$ staining using an anti-luciferase antibody $(n=12$ per group). Scale bars: $500 \mu \mathrm{m}$ (black); $50 \mu \mathrm{m}$ (red). metastasis-free survival (Figure 1C and Supplemental Figure 1D), suggesting a potential link between a high BLACAT2 expression level and human bladder cancer progression.

Interestingly, analyses of TCGA and GEO databases showed that BLACAT2 expression was also significantly upregulated in multiple types of human cancer, such as lung cancer, thyroid cancer, liver cancer, and glioma (Supplemental Figure 2, A-G), and higher expression of BLACAT2 correlated with poor prognosis in glioma (Supplemental Figure 2H), further supporting the oncogenic role of BLACAT2 in cancer.

BLACAT2 is located on human chromosome 13p15.2 (Supplemental Figure 3A). In our assessment, the full-length BLACAT2 transcript was $1120 \mathrm{nt}$ in the bladder cancer cell lines, which were examined using the $5^{\prime}$ and $3^{\prime}$ rapid amplification of cDNA end (RACE) method (Supplemental Figure 3, B and C).

Consistent with the results obtained by RT-qPCR, an in situ hybridization (ISH) analysis showed that BLACAT2 expression was mildly detectable in normal bladder tissues and moderately expressed in non-LN-metastatic bladder cancer tissues; however, BLACAT2 was strongly upregulated in LN-metastatic bladder cancer (Supplemental Figure 3D). Both the ISH analyses and the subcellular fractionation assay indicated that BLACAT2 mainly localized to the nuclei of bladder cancer cells (Supplemental Figure $3, \mathrm{E}$ and $\mathrm{F}$ ).

BLACAT2 level correlates with the intratumoral and peritumoral lymphatic vessel density. Tumor-associated lymphangiogenesis, which is an independent prognostic factor in bladder cancer, is associated with LN metastasis $(12,29)$. Importantly, statistical analysis revealed that BLACAT2 expression was significantly correlated with microlymphatic vessel density (MVD), as indicated by LYVE-1-positive microvessels in both the intratumoral and the peritumoral regions of bladder cancers $(P<0.001$ and $P<0.001$, respectively), suggesting that BLACAT2 may play a vital role in lymphangiogenesis in bladder cancer (Figure 1, D and E).

$B L A C A T 2$ promotes $L N$ metastasis in vivo. To investigate the role of BLACAT2 in LN metastasis, UM-UC-3/luc and HT-1376/ luc bladder cancer cell lines were established to stably overexpress BLACAT2 or an shRNA targeting BLACAT2 (Supplemental Figure 4, A and B). These cells were implanted into the footpads of nude mice (Supplemental Figure 4C). Strikingly, the volumes of the popliteal LNs were dramatically larger in the BLACAT2/ mice, but smaller in the BLACAT2 shRNA/mice, than those in the corresponding control mice (Figure 2A and Supplemental Figure 5A). Immunostaining of luciferase confirmed that forced 
A

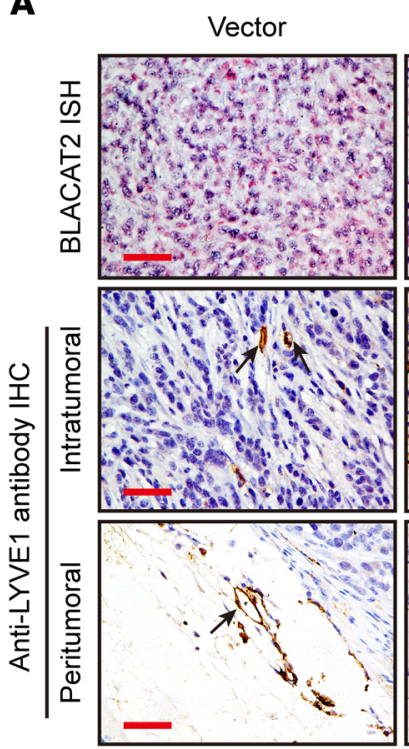

B
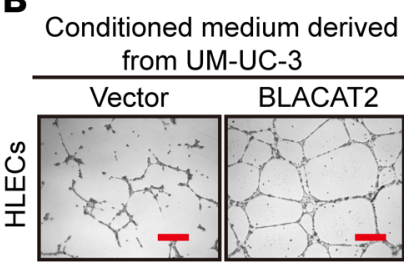

Conditioned medium derived from 5637

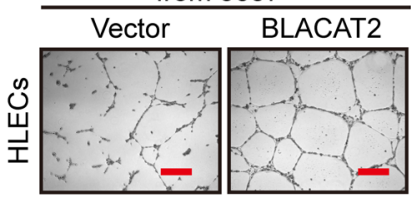

BLACAT2
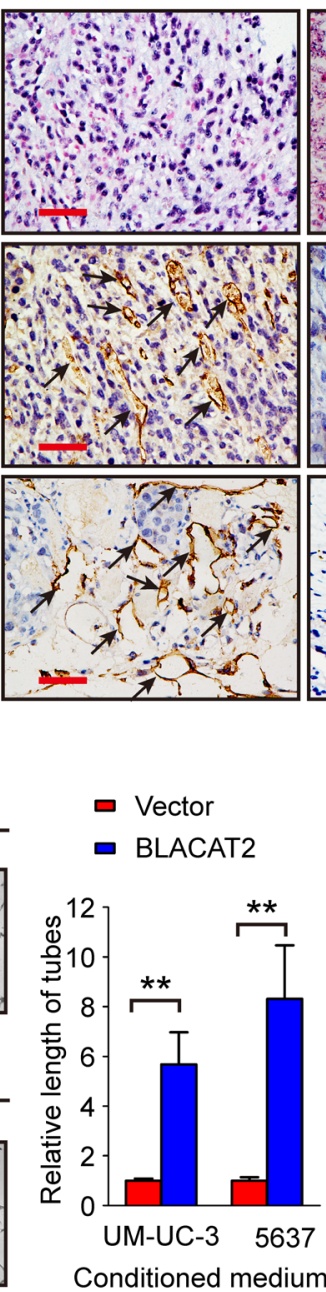

shControl
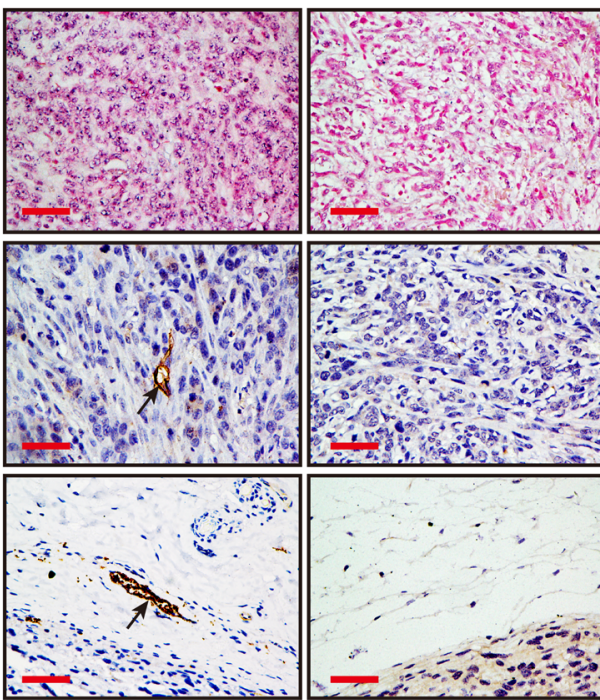

C

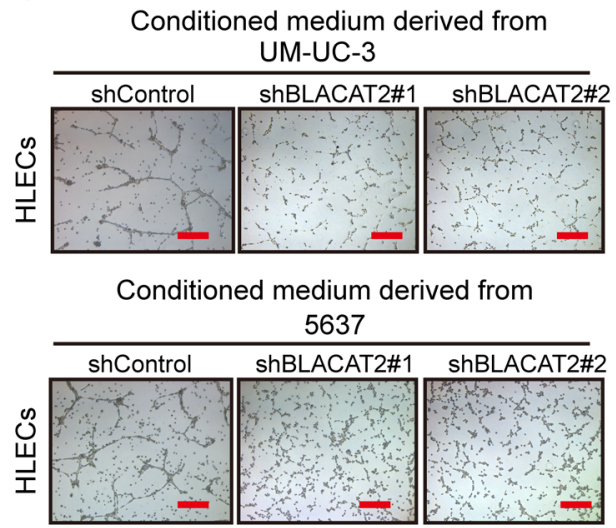

$\begin{array}{ll}\text { - Vector } & \text { shControl } \\ \text { - BLACAT2 } & \text { a shBLACAT2\#1 }\end{array}$

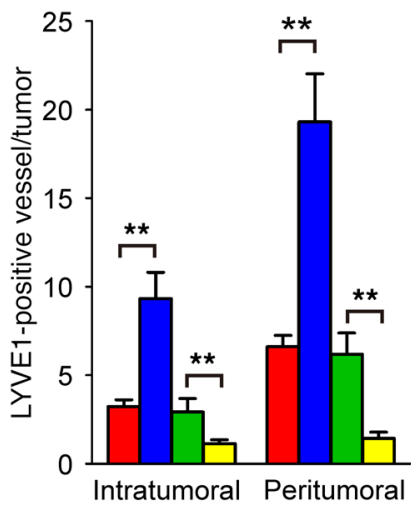

a shControl

- shBLACAT2\#1

口 shBLACAT2\#2

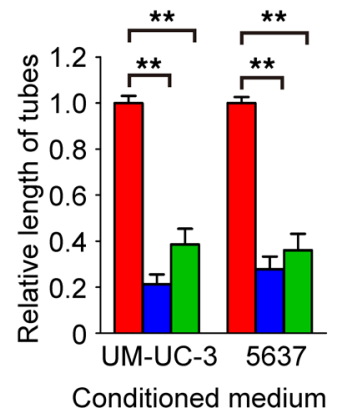

Figure 3. BLACAT2 overexpression promotes lymphangiogenesis in vitro and in vivo. (A) Representative images of intratumoral and peritumoral microlymphatic vessels stained with anti-LYVE1 (left panel) and histogram of the quantification of microlymphatic vessel density (right panel) ( $n=12$ ). BLACAT2 expression levels were confirmed by ISH. Scale bars: $50 \mu \mathrm{m}$. (B and C) Representative images (left panels) and histogram quantification (right panels) of the Matrigel tube formation assay with HLECs. HLECs were cultured with conditioned medium derived from bladder cancer cells that were treated as indicated. Scale bars: $200 \mu \mathrm{m}$. All experiments in vitro were performed with at least 3 biological replicates. The error bars indicate the SD of the mean. Statistical significance was assessed using 2-tailed Student's $t$ test ( $\mathbf{A}$ and $\mathbf{B})$ and 1-way ANOVA followed by Dunnett's tests for multiple comparisons (C). ${ }^{*} P<0.01$.

expression of BLACAT2 significantly promoted the metastatic capability of bladder cancer cells to LNs and that ablation of BLACAT2 inhibited LN metastasis (Figure 2B and Supplemental Figure 5B), as determined by the number of metastatic LNs (Supplemental Figure 6A). The tumor/BLACAT2-bearing mice had shorter survival times, and the tumor/BLACAT2 shRNAbearing mice had longer survival times (Supplemental Figure 6B). Meanwhile, we observed only small volume alterations of xenografted tumors in the footpads of nude mice between mice with high BLACAT2 expression and those with low BLACAT2 expression (Supplemental Figure 7A), and the number and distribution of metastatic foci in lung were higher in BLACAT2-overexpressing groups than in the control group, suggesting that the reason BLACAT2-overexpressing mice died earlier was distant metastasis (Supplemental Figure 7B). Taken together, these results indicate that BLACAT2 plays a critical role in LN metastasis of bladder cancer in vivo.
BLACAT2 induces lymphangiogenesis in vivo and in vitro. Because lymphangiogenesis is a rate-limiting step for $\mathrm{LN}$ metastasis of bladder cancer $(10,11)$, we further examined whether BLACAT2 overexpression promotes lymphangiogenesis in the animal model. Importantly, the quantity of intratumoral and peritumoral lymphatic vessels in the primary tumors, which were assessed using an antibody to a lymphatic marker, LYVE-1, were dramatically increased in mice bearing the BLACAT2-transduced cells and decreased in the mice transplanted with BLACAT2-silenced cells (Figure 3A), indicating that BLACAT2 promotes lymphangiogenesis in vivo.

Next, we further evaluated the effect of BLACAT2 overexpression on the promotion of lymphangiogenesis in vitro. Tube formation assays showed that the conditioned medium from the BLACAT2-transduced bladder cells strongly provoked tube formation by human lymphatic endothelial cells (HLECs), whereas BLACAT2 silencing abolished the ability of bladder cancer cells 
A

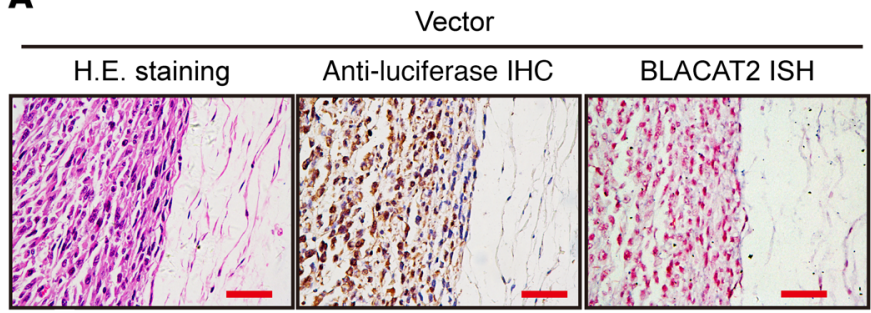

B
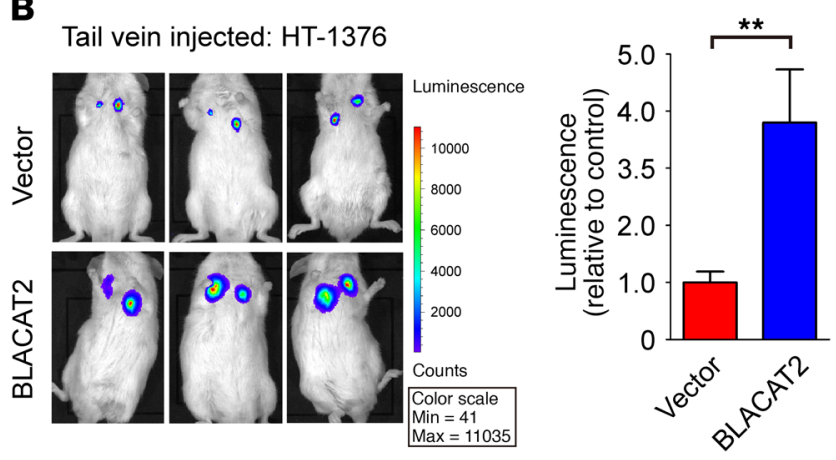

D

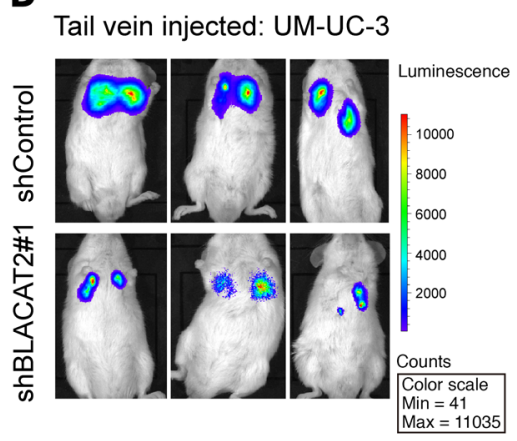

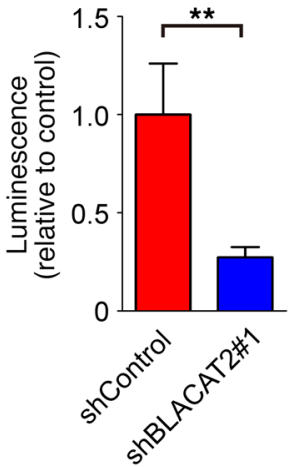

BLACAT2

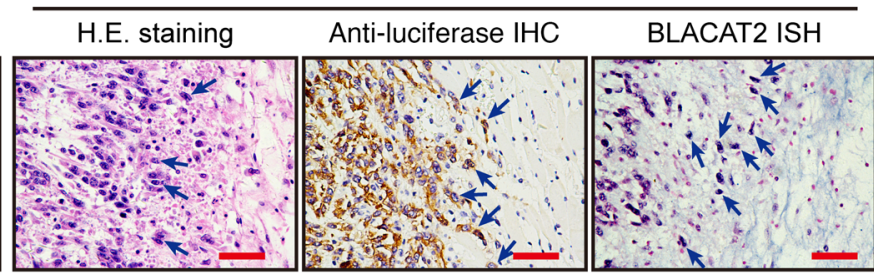

C
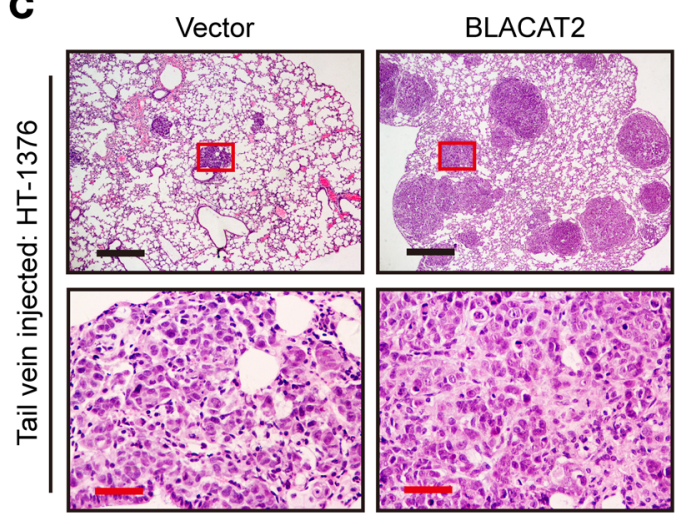

E

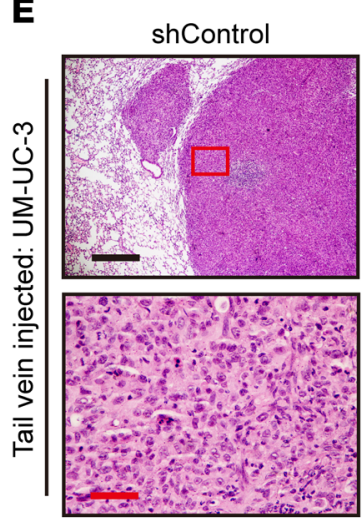

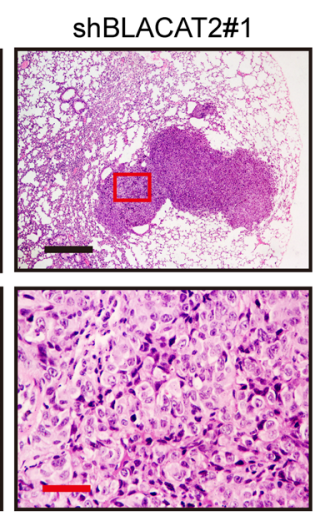

Figure 4. BLACAT2 overexpression promotes bladder cancer cell invasion and metastasis in vivo. (A) Representative images of H\&E staining, IHC staining with antiluciferase, and BLACAT2 ISH staining for visualization of human bladder cancer cells invading surrounding tissues in vivo, as indicated by arrows $(n=12)$. Scale bars: $50 \mu \mathrm{m}$. (B-E) Representative images of lung colonization by bladder cancer cells injected into tail veins of NOD/SCID mice (B and $\mathbf{D}$, left panels) and histogram analysis of luminescence representing lung metastasis measured on day 40 (B and $\mathbf{D}$, right panels). $n=12$. Lung metastasis was confirmed by H\&E staining (C and E). Cells were transduced with vectors as indicated. Scale bars: $200 \mu \mathrm{m}$ (black); $50 \mu \mathrm{m}$ (red). Error bars indicate the SD of the mean. Statistical significance was assessed using 2-tailed Student's $t$ test (B and $\mathbf{D}) .{ }^{* *} P<0.01$.

to induce HLEC tube formation (Figure 3, B and C). Additionally, Transwell migration assays revealed that the migratory speed of HLECs was dramatically increased in the conditioned medium of BLACAT2-transduced cells, but decreased in the BLACAT2silenced conditioned medium (Supplemental Figure 8, A and B). Therefore, our results suggest that BLACAT2 overexpression induces lymphangiogenesis in vivo and in vitro.

BLACAT2 promotes metastasis of bladder cancer cells. LN metastasis is a complex multistep process (30). In addition to intratumoral and peritumoral lymphangiogenesis, enhanced cell invasion and extracellular matrix degradation are essential for metastasis $(31,32)$. Importantly, we noticed that the tumors formed by the BLACAT2transduced bladder cancer cells grown in the footpads of the nude mice displayed spike-like structures that invaded the surrounding tissues, while the control tumors exhibited sharp edges (Figure 4A), suggesting that overexpression of BLACAT2 may augment the invasive ability of bladder cancer cells.

Next, wound healing and Transwell cell migration/invasion assays revealed that ectopic expression of BLACAT2 significantly enhanced the motility (Supplemental Figure 9, A-D) and invasiveness (Supplemental Figure 10, A and B) of bladder cancer cells, whereas the opposite outcome was observed after BLACAT2 depletion. Moreover, 3D epithelial Matrigel culture models, which mimic the process of tumor invasion of the basement membrane, showed that BLACAT2 overexpression accelerated bladder cancer cell invasion, whereas BLACAT2 ablation drastically suppressed the invasive capability of the bladder cancer cells (Supplemental Figure 10C). These results also suggested that BLACAT2 may 
A

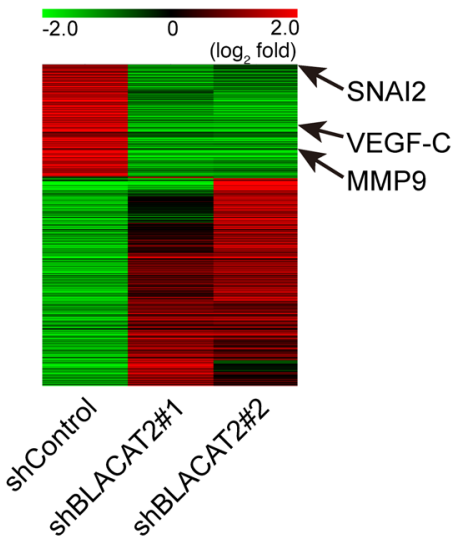

B

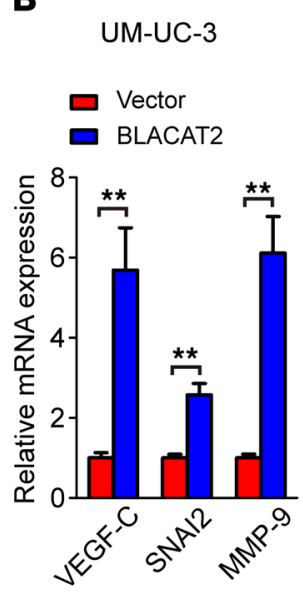

C

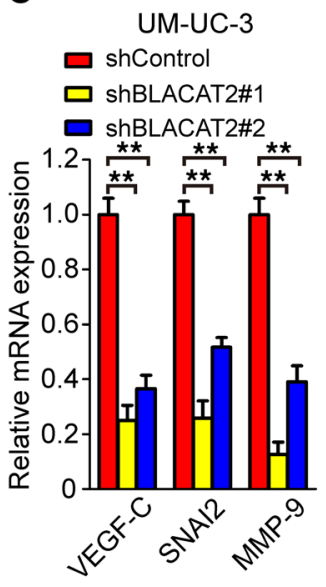

D

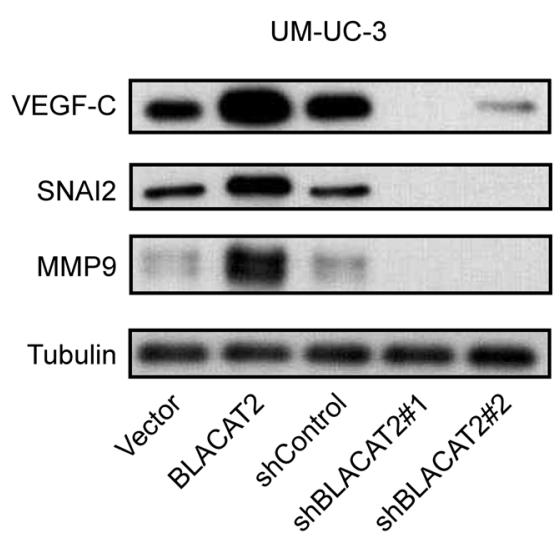

E
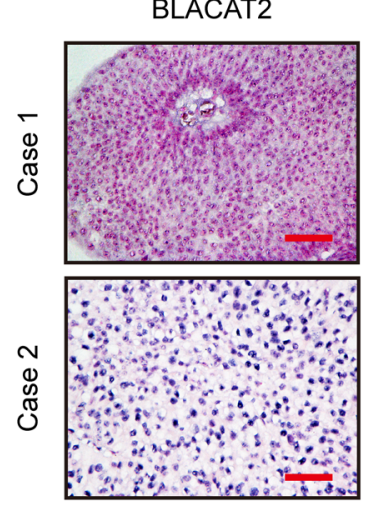

VEGF-C

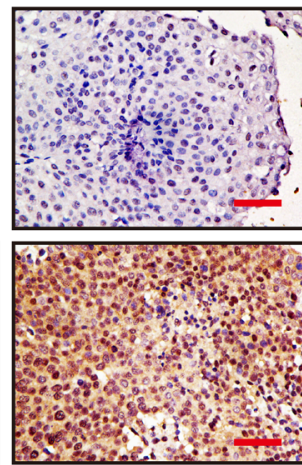

SNAI2
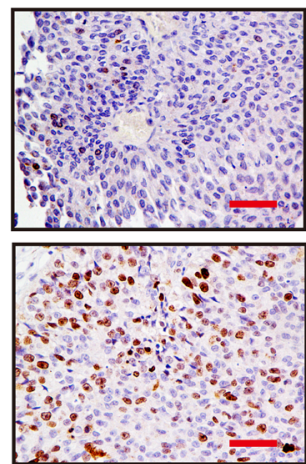

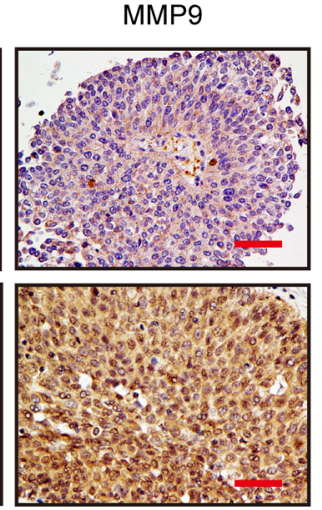

Spearman $r=0.722 \quad P<0.001$ Kendall $\mathrm{r}=0.592 \quad P<0.001$

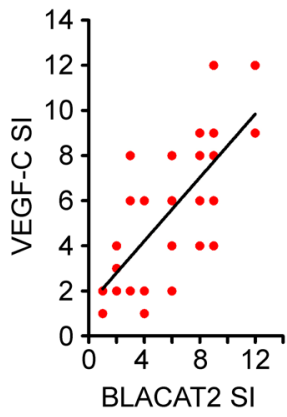

Figure 5. BLACAT2 regulates VEGF-C, SNAI2, and MMP9 expression. (A) Heatmap representing unsupervised hierarchical clustering of genes regulated by BLACAT2 quantified by NGS. Rows represent probe sets, and columns represent samples treated as indicated. Green, downregulation; red, upregulation. SNAI2, VEGF-C, and MMP9 are indicated by black arrows. (B and C) Bladder cancer cells were transduced with a lentivirus-based BLACAT2-overexpressing vector (B), BLACAT2 shRNAs (C), or control vectors as indicated. Transduced cells were harvested 96 hours later, and VECF-C, SNAI2, and MMP9 mRNA expression levels were quantified by RT-qPCR. Statistical significance was assessed using 2-tailed Student's $t$ test (B) and 1-way ANOVA followed by Dunnett's tests for multiple comparisons (C) $\left(n=3,{ }^{* *} P<0.01\right)$. (D) Representative image of the Western blotting analysis of VEGF-C, SNAI2, and MMPg protein levels after BLACAT2 overexpression or depletion in UM-UC-3. (E) Representative images (left panels) and correlation analysis (right panel) of IHC staining showing that BLACAT2 expression positively correlates with VEGF-C, SNAI2, and MMPg expression levels in the bladder cancer tissues. $n=140$. Scale bars: $50 \mu \mathrm{m}$.

be involved in the EMT of bladder cancer cells. As expected, the RT-qPCR, immunoblotting, and immunofluorescence analyses revealed that E-cadherin, an epithelial marker, was downregulated, whereas the expression levels of $\mathrm{N}$-cadherin, vimentin, and fibronectin, which are mesenchymal markers, were increased in BLACAT2-overexpressing cells (Supplemental Figure 11, A-F). Consistently, BLACAT2 silencing dramatically reversed the TGF- $\beta$-induced mesenchymal feature of the UM-UC-3 and 5637 bladder cancer cell lines (Supplemental Figure 11, B-F). Furthermore, we found that depletion of BLACAT2 in UM-UC-3 cells, which represents a mesenchymal phenotype at baseline, significantly downregulated mesenchymal biomarkers, such as fibronectin and vimentin (Supplemental Figure 12, A and B).

Additionally, tail-vein assays showed that BLACAT2 overexpression expanded the metastatic bladder cancer cell colonization of the lung (Figure 4, B and C), whereas BLACAT2 silencing strongly decreased lung colonization by tumor cells (Figure 4, D and E). Collectively, these results indicate that BLACAT2 increases the invasiveness of bladder cancer cells both in vitro and in vivo.
BLACAT2 regulates VEGF-C, SNAI2, and MMP9 expression. To explore the molecular mechanisms underlying BLACAT2-induced lymphatic metastasis in bladder cancer, we profiled BLACAT2silenced UM-UC-3 cells and control cells using next-generation sequencing (NGS). Among 1,384 genes that were regulated by BLACAT2 $(P<0.05$, fold changes $>2.0)$, multiple genes that play critical roles in lymphatic metastasis, such as VEGF-C, SNAI2, and MMP9 (33-35), were significantly downregulated in BLACAT2silenced cells (Figure 5A). Furthermore, immunostaining and qPCR analyses revealed that the expression of VEGF-C, SNAI2, and MMP9, at both the mRNA and the protein levels, was increased in BLACAT2-transduced cells and decreased in BLACAT2-silenced cells (Figure 5, B-D, and Supplemental Figure 13, A-E). Furthermore, BLACAT2 expression levels correlated with VEGF-C expression in human bladder cancer cell lines (Supplemental Figure 14A). Moreover, positive correlations between BLACAT2 expression and the VEGF-C, SNAI2, and MMP9 levels $(P<0.001, P<0.001, P<$ 0.001 , respectively) were also observed in the xenografts (Supplemental Figure 14, B and C) and in a 140-case cohort of bladder 
A
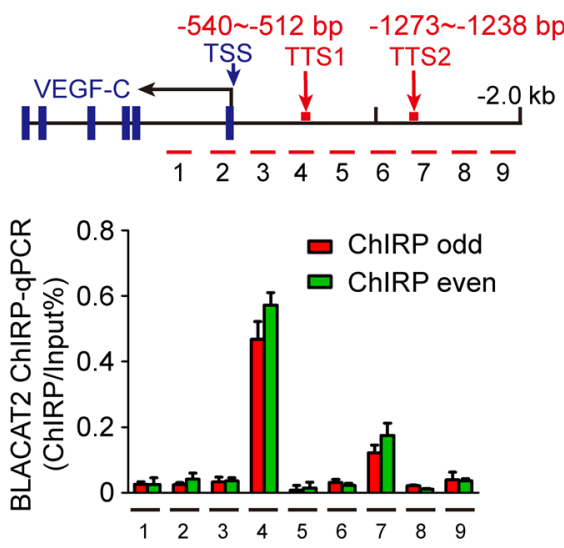

B

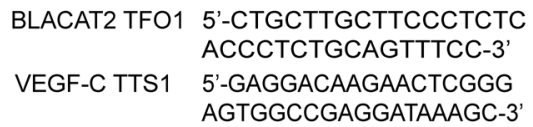

BLACAT2 TFO1 + VEGF-C TTS1

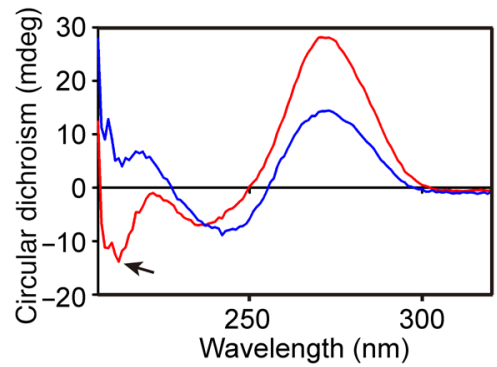

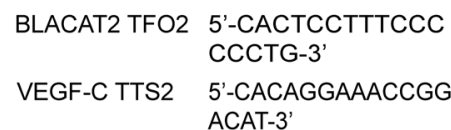

BLACAT2 TFO2 + VEGF-C TTS2

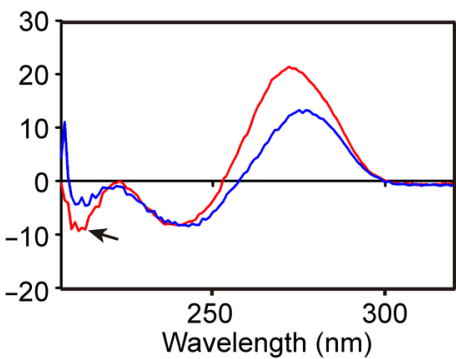

C

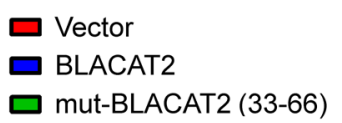

D

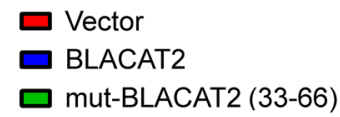

E

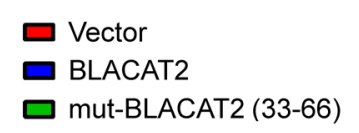

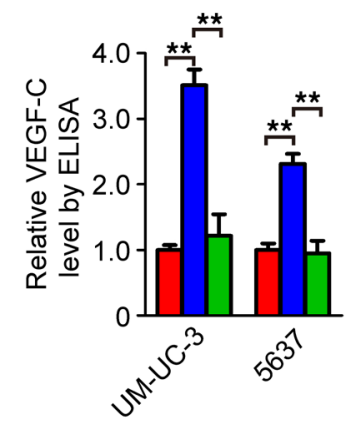

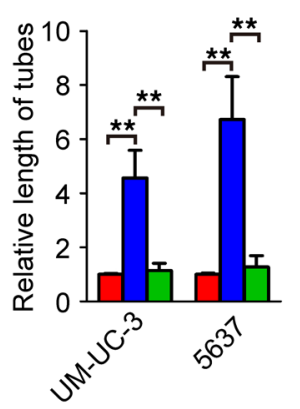

Conditioned medium

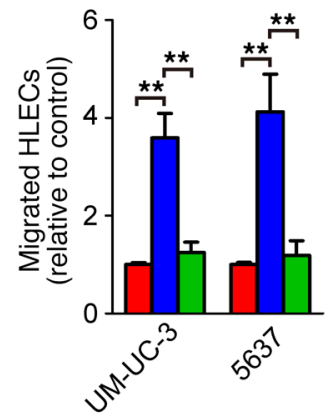

Conditioned medium

Figure 6. BLACAT2 directly binds to VEGF-C promoter sequences. (A) ChIRP analysis of BLACAT2-associated chromatin in UM-UC-3 cells. Retrieved chromatin was quantified by PCR. The percentage recovery of input for ChIRP was calculated based on a 10\% nonprecipitated DNA sample for each experiment. The blue arrow indicates the transcriptional start site (TSS). The red arrows indicate the location of transcriptional start sites. (B) CD spectrum of a 1:1 mixture of TFO in BLACAT2 with TTS in the VEGF-C promoter sequences is shown in red. The sum of individual TFO and TTS is shown in blue. (C) Site-directed mutagenesis of 33-66 nt in BLACAT2 was performed, and the effect of overexpression of the wild-type or mutated BLACAT2 on VEGF-C secretion was evaluated by ELISA. (D and E) The effects of overexpression of wild-type or mutated BLACAT2 on tube formation (D) and migration of HLECs (E) were evaluated. All experiments were performed with at least 3 biological replicates. Statistical significance was assessed using 1-way ANOVA followed by Dunnett's tests for multiple comparisons $(\mathbf{C}-\mathbf{E}) .{ }^{*} P<0.01$.

cancer specimens (Figure 5E and Supplemental Figure 14D). These results indicate that BLACAT2 contributes to the upregulation of VEGF-C, SNAI2, and MMP9 in bladder cancer.

BLACAT2 forms triplexes with promoter sequences of VEGF-C. Next, we focused on the molecular mechanisms of BLACAT2 in the expression of VEGF-C and performed an RNA purification assay (chromatin isolation by RNA purification [ChIRP]) to identify whether BLACAT2 directly associates with promoter sequences of VEGF-C. The ChIRP assay indicated that BLACAT2 could physiologically associate with the promoter sequences of VEGF-C (Figure 6A). To further identify the more precise direct binding sites between BLACAT2 and VEGF-C promoter, potential triplexforming oligos (TFOs) and corresponding triplex target sites (TTSs) were predicted with Longtarget, a web-based tool designed to predict lncRNA:DNA binding motifs and binding sites via Hoogsteen or reverse Hoogsteen base pairing (36). Circular dichroism (CD) spectroscopy was performed using in vitro-synthesized predicted TFOs of BLACAT2 and TTSs of the VEGF-C promoter. The spectrum of BLACAT2 (33-66 nt)/VEGF-C TTS1 or TTS2 showed an obvious negative peak at approximately $210 \mathrm{~nm}$ and a positive peak at 270-280 nm (Figure 6B and Supplemental Figure 15A) when compared with the FENDRR/PITX2-positive control group and the ssRNA/PITX2-negative control group (Supplemental Figure 15, B and C) (37), suggesting that BLACAT2 (33-66 nt) formed triplexes with 2 binding sites of promoter sequences of VEGF-C in vitro. Moreover, BLACAT2 that was mutated at 33-66 nt failed to induce VEGF-C expression or promote HLEC tube formation and migration (Figure 6, C-E, and Supplemental Figure 16, A-D). In addition, silencing of VEGF-C in BLACAT2-transduced cells only slightly decreased the expression levels of SNAI2 and MMP9, suggesting that BLACAT2-induced upregulation of SNAI2 and MMP9 did not rely on VEGF-C (Supplemental Figure 17, A and B).Taken together, these data indicate that BLACAT2 directly forms triplexes with the promoter sequences of VEGF-C.

BLACAT2 directly binds to the WDR5 protein. Next, we performed RNA pull-down assay using biotin-labeled BLACAT2 as 
A

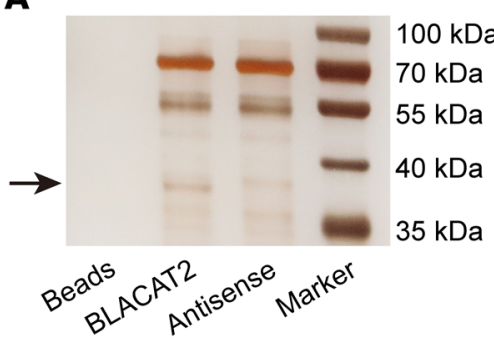

B

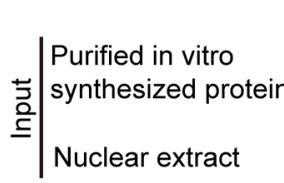

WDR5 blot after RNA pull-down assay

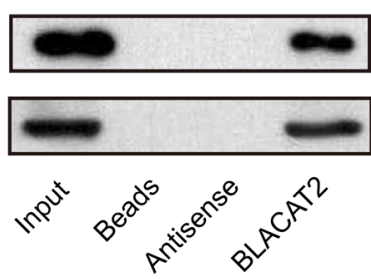

C

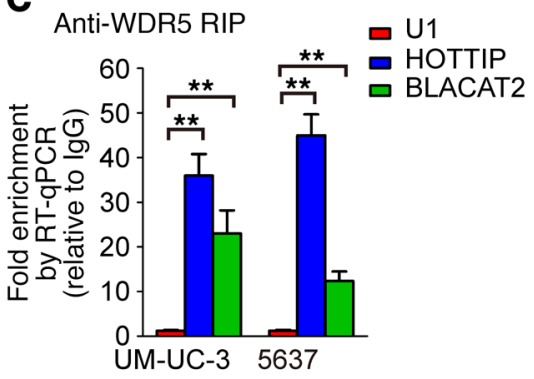

D

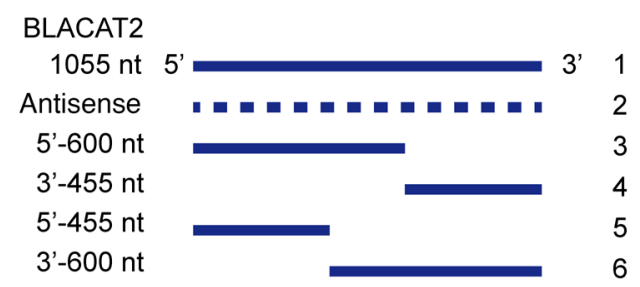

WDR5

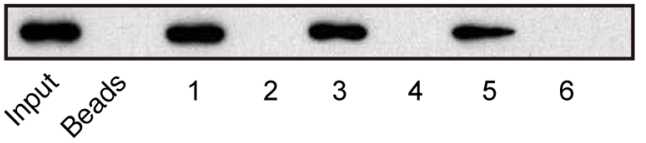

$\mathbf{F}$

$\square$ Vector

BLACAT2

$\square$ mut-BLACAT2 (100-130)

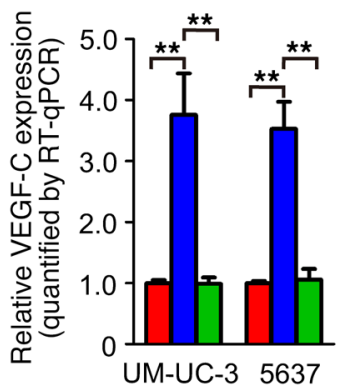

G

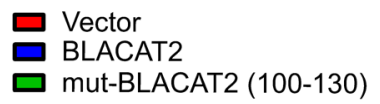

$\square$ mut-BLACAT2 (100-130)

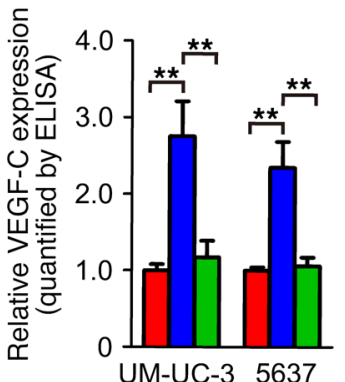

E

BLACAT2

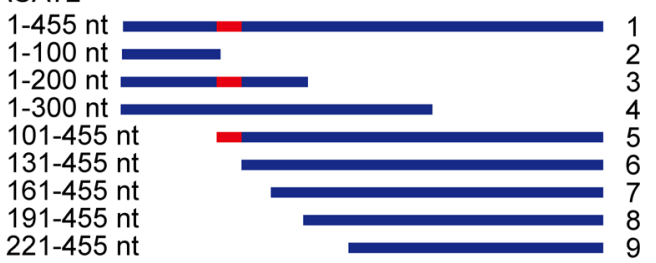

WDR5
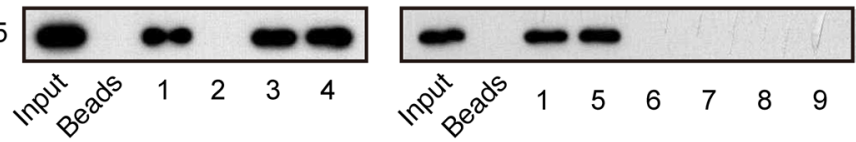

H
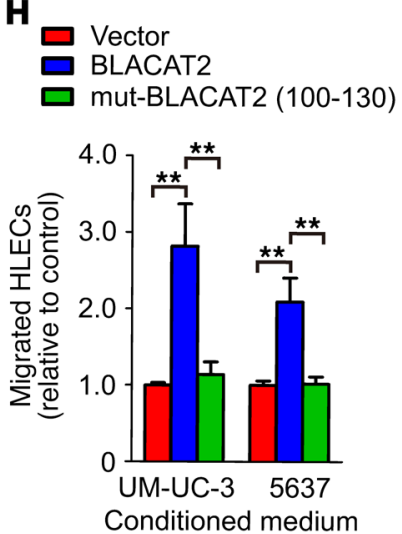

1

$\square$ Vector

BLACAT2

$\square$ mut-BLACAT2 (100-130)

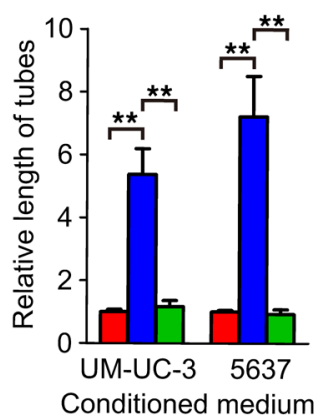

Figure 7. BLACAT2 directly binds to WDR5 protein and regulates VEGF-C expression. (A) Representative image of silver-stained PACE gels showing separated proteins that were pulled down using biotin-labeled BLACAT2. In vitro-transcribed antisense sequence of BLACAT2 was used as the nonspecific control. (B) Western blot analysis indicating that BLACAT2 associates with WDR5, as indicated by the pull-down assay with nuclear extracts or in vitrosynthesized WDR5. Antisense BLACAT2 was used as the negative control RNA in the pull-down assay. (C) RT-qPCR analysis of RNA enrichment in the RIP assay using the anti-WDR5 antibody in UM-UC-3 and 5637 bladder cancer cells. Normal IgG was used as the nonspecific control antibody. U1 and HOTTIP were used as negative and positive controls, respectively, for WDR5 binding. (D and E) Serial deletions of BLACAT2 were used in RNA pull-down assays to identify core regions of BLACAT2 that were required for physical interaction with WDR5. (F-I) Site-directed mutagenesis of 100-130 nt of BLACAT2 was performed, and the effects of BLACAT2 mutant overexpression on VEGF-C mRNA expression (F), VECF-C secretion (G), HLEC migration (H), and HLEC tube formation (I) were evaluated. All experiments were performed with at least 3 biological replicates. Statistical significance was assessed using 1 -way ANOVA followed by Dunnett's tests for multiple comparisons (C, F-I). ${ }^{* *} P<0.01$.

bait to identify BLACAT2-interacting proteins in UM-UC-3 bladder cancer cells. As shown in Figure 7A, an evident band with a molecular weight between 35 and $40 \mathrm{kDa}$ was subjected to mass spectrometry (MS), which highlighted WDR5, a core component of the histone $\mathrm{H} 3 \mathrm{~K} 4$ methyltransferase complex, as a potent BLACAT2-interacting protein (Supplemental Figure 18). Furthermore, both in vivo and in vitro RNA immunoprecipitation (RIP) assays revealed that BLACAT2 could interact with endogenous WDR5 in bladder cancer cells (Figure 7B) and with recombinant WDR5 protein (Figure $7 \mathrm{C}$ ), indicating that BLACAT2 directly binds to WDR5. Moreover, a serial deletion analysis revealed that nt 100-
130 in the $5^{\prime}$ terminal of the BLACAT2 transcript are critical for the interaction with WDR5 (Figure 7, D and E). Consistently, forced expression of the mutant BLACAT2, which had a 100-130 nt deletion in its $5^{\prime}$ terminal, failed to induce VEGF-C expression, HLEC migration, and tube formation (Figure 7, F-I). However, overexpression of $5^{\prime}$ terminal-truncated BLACAT2 (1-200 nt) in BLACAT2-silenced cancer cells restored the function of BLACAT2 in VEGF-C expression regulation, HLEC migration, and HLEC tube formation (Figure 8, A-D). These results demonstrate that the $5^{\prime}$ terminal region of BLACAT2 is critical for the interaction with WDR5 and for the regulation of VEGF-C expression. 
A

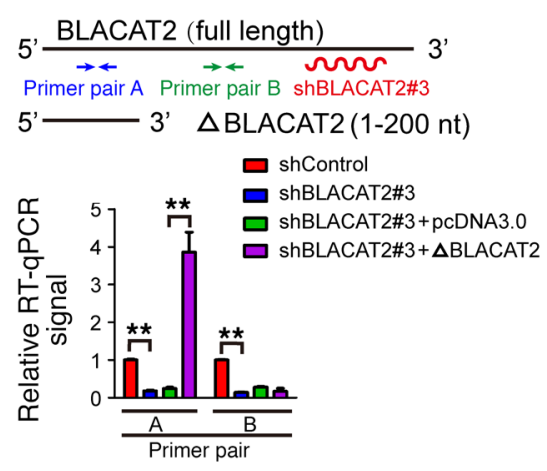

B

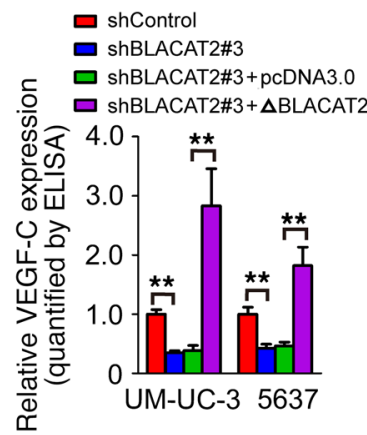

C

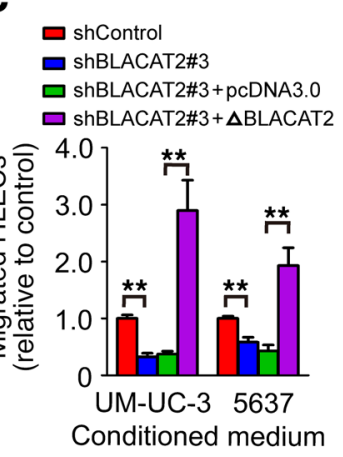

D

$$
\square \text { shControl }
$$

ShBLACAT2\#3

口

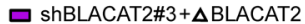

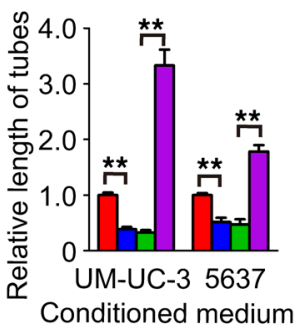

E

$$
\begin{aligned}
& \text { UM-UC-3 } \\
& \text { Vector } \\
& \text { ․ BLACAT2 } \\
& \text { mut-BLACAT2 (33-66) }
\end{aligned}
$$

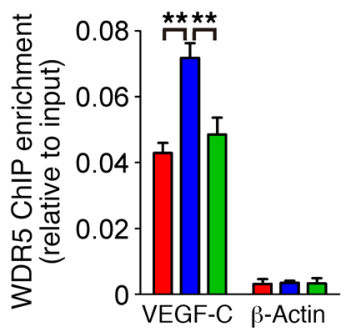

$\mathbf{F}$

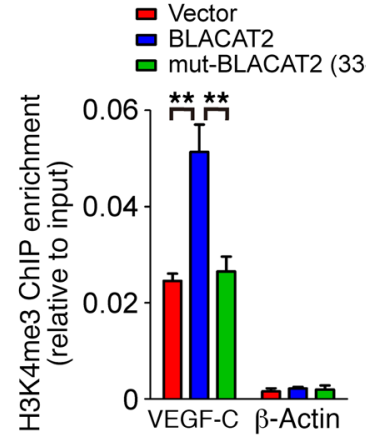

G

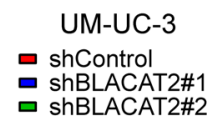

H

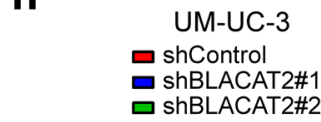

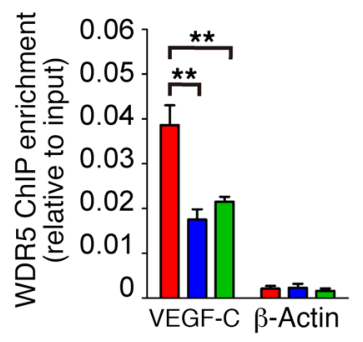

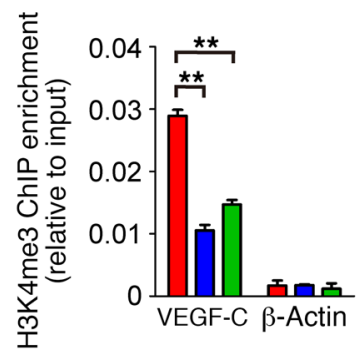

Figure 8. BLACAT2 recruits WDR5 protein and modulates H3K4 trimethylation of VEGF-C promoter. (A) Endogenous BLACAT2 was depleted with an shRNA that targeted the 3 ' terminal site, and the efficiency of forced expression of the truncated BLACAT2 (1-200 nt) was examined by RT-qPCR. (B-D) The effects of truncated BLACAT2 on VEGF-C secretion (B), HLEC migration (C), and HLEC tube formation (D) were evaluated. (E-H) ChIP-qPCR analysis of the WDR5 genomic occupancy (E and $\mathbf{G}$ ) and H3K4 methylation status ( $\mathbf{F}$ and $\mathbf{H})$ in the VEGF-C promoter after overexpression $(\mathbf{E}$ and $\mathbf{F})$ or depletion $(\mathbf{G}$ and $\mathbf{H})$ of BLACAT2 in UM-UC-3 cells as indicated. All experiments were performed with at least 3 biological replicates. Error bars indicate SD of the mean. Statistical significance was assessed using 2-tailed Student's $t$ test (B-D) and 1-way ANOVA followed by Dunnett's tests for multiple comparisons $(\mathbf{E}$ and $\mathbf{H}) .{ }^{* *} P<0.01$.

BLACAT2 promotes H3K4 methylation of promoters of downstream target genes. WDR5, a core unit of human H3K4 methyltransferase complexes (38), epigenetically modulates target gene expression by associating with methylated $\mathrm{H} 3 \mathrm{~K} 4$ to catalyze $\mathrm{H} 3 \mathrm{~K} 4$ trimethylation (39). We sought to determine whether BALCAT2 regulated VEGF-C via WDR5-mediated H3K4 methylation. ChIP showed that BLACAT2 overexpression dramatically enhanced WDR5 expression and increased $\mathrm{H} 3 \mathrm{~K} 4 \mathrm{me} 3$ methylation of the promoters of VEGF-C both in WDR5 $5^{\text {hi }}$-expressing UM-UC-3 or 5637 cells and WDR5 $5^{\text {lo }}$ expressing T24 and RT4 cells, whereas BLACAT2 silencing drastically decreased WDR5 expression and H3K4me3 methylation of the promoters of VEGF-C (Figure 8, E-H, and Supplemental Figure 19, A-E). Additionally, WDR5 silencing attenuated BLACAT2-induced expression of VEGF-C- and BLACAT2-mediated lymphangiogenesis (Supplemental Figure 20, A-E). Importantly, in SV-HUC-1 cells, an immortalized normal bladder epithelial cell line, we also observed the same effects of BLACAT2 on the upregulation of VEGF-C expression, promoting the recruitment of WDR5 and promotion of $\mathrm{H} 3 \mathrm{~K} 4$ trimethylation of the VEGF-C promoter and provoking the tube formation and migratory speed of HLECs (Supplemental Figure $21, \mathrm{~A}-\mathrm{F})$. These results suggest that BLACAT2 might also play a role in regulating physiological lymphangiogenesis, and this mechanism was not exclusive to tumors. Therefore, our results indicate that BLACAT2 promotes H3K4 methylation of the VEGF-C promoter.
Similarly, overexpression of BLACAT2 significantly increased, but downregulation of BLACAT2 decreased, WDR5 occupancy and $\mathrm{H} 3 \mathrm{~K} 4$ trimethylation status on SNAI2 and MMP9 promoters, whereas mut-BLACAT2 (33-66 nt) failed to induce WDR5 occupancy and H3K4 trimethylation (Supplemental Figure 22, A and B). ChIRP analysis indicated that BLACAT2 associates with the promoter sequences of SNAI2 and MMP9 (Supplemental Figure 22C).

$V E G F-C$ is required for BLACAT2-induced lymphangiogenesis and LN metastasis. Accumulating evidence has been provided that VEGF-C signaling, which plays critical roles in lymphangiogenesis and LN metastasis, may serve as a potential therapeutic target for $\mathrm{LN}$-metastatic cancer $(32,34,40,41)$. We tested to determine whether downregulation of VEGF-C could inhibit BLACAT2induced LN metastasis. VEGF-C silencing showed more inhibitory effects on LN metastasis of BLACAT2-transduced cells relative to control cells (Figure 9, A and B, and Supplemental Table 3), resulting in longer survival times for the BLACAT2/mice relative to control mice (Supplemental Figure 23, A and B). This result was further confirmed by the higher intratumoral and peritumoral lymphangiogenesis observed in the BLACAT2/mice relative to the control mice (Figure 9C). Consistently, knockdown of VEGF-C significantly inhibited BLACAT2-induced HLEC tube formation and migration, and overexpression of VEGF-C rescued the effects of BLACAT2 ablation on HLEC tube formation and migration (Supplemental 
A

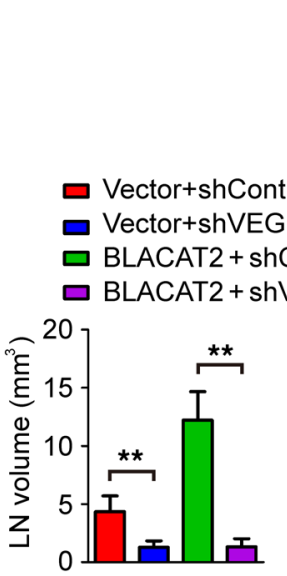

B
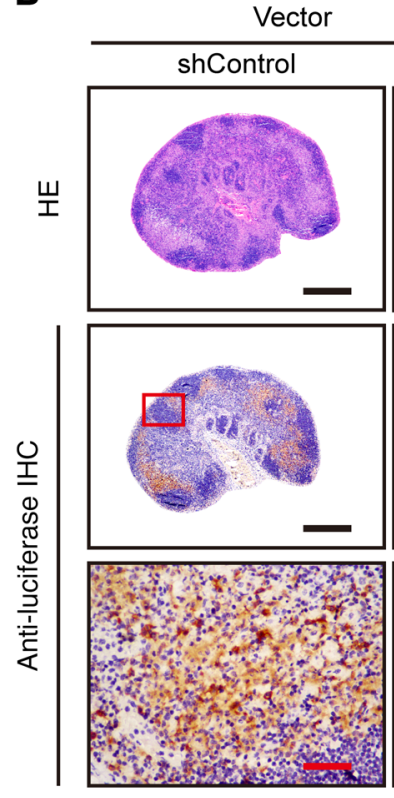
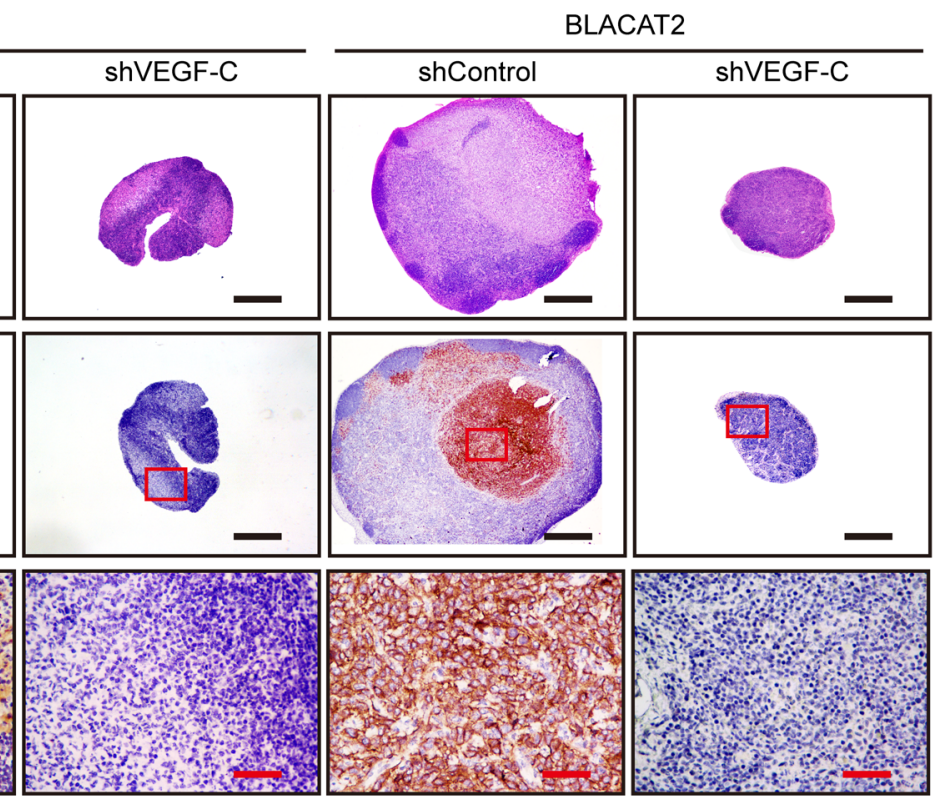

C

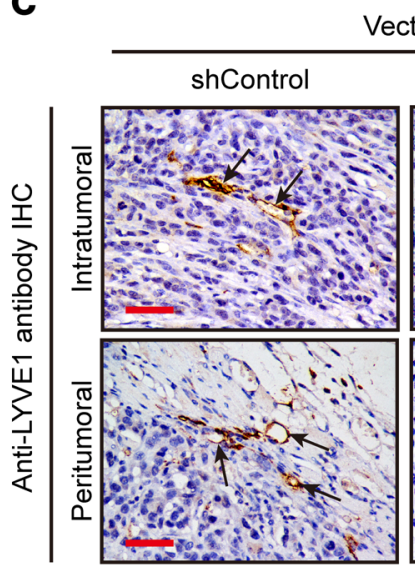

Vector

BLACAT2
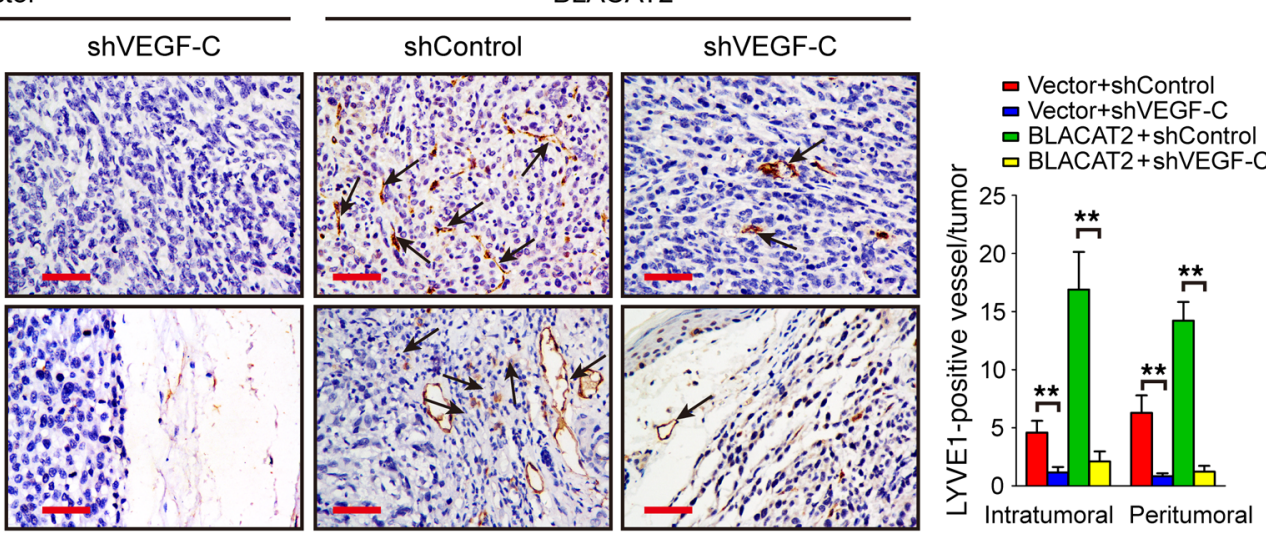

Figure 9. Depletion of VEGF-C abrogates BLACAT2-induced LN metastasis in vivo. (A) Volume quantification of popliteal LN metastasis after shRNAmediated depletion of VEGF-C. Popliteal LNs were enucleated and analyzed at the time of death or after 60 days ( $n=12$ per group). (B) Representative images of H\&E staining and IHC staining confirming LN status $(n=12)$. Scale bars: $500 \mu \mathrm{m}$ (black); $50 \mu \mathrm{m}$ (red). (C) Representative images of intratumoral and peritumoral microlymphatic vessels stained with anti-LYVE1 (left panel, as indicated with black arrows) and histogram quantification of microlymphatic vessel density (right panel). Error bars represent SD of the mean. ${ }^{* *} P<0.01$, Student's $t$ test (A and $\left.\mathbf{C}\right)$. Scale bars: $50 \mu \mathrm{m}$.

Figure 24, A-D). However, silencing of SNAI2 only slightly reduced the metastatic capability of bladder cancer cells to LNs (Supplemental Figure 25, A-D), suggesting that VEGF-C is essential for BLACAT2-induced lymphangiogenesis and LN metastasis.

Blockage of VEGF-C reverses BLACAT2-induced LN metastasis in vivo. The VEGF-C monoclonal antibody (VGX-100) has been tested in a clinical trial for advanced or metastatic solid tumors. We examined whether VEGF-C signaling blockade using a VEGF-C-neutralizing antibody (pV1006R-r) could inhibit BLACAT2-induced LN metastasis. Similar to the effect of VEGF-C silencing, treatment with the VEGF-C-neutralizing antibody (pV1006R-r) significantly reduced BLACAT2-induced HLEC tube formation and motility (Supplemental Figure 26, A and B), suggesting that deactivation of VEGF-C signaling could inhibit BLACAT2-mediated lymphangiogenesis in vitro. The thera- peutic effect of targeting VEGF-C was then further examined in vivo. A popliteal LN metastasis model showed that treatment with the VEGF-C-neutralizing antibody (pV1006R-r) led to a significant reduction in intratumoral and peritumoral lymphatic vessel densities and decreased BLACAT2-transduced tumor burden in the LNs (Figure 10, A-C, and Supplemental Table 3), which lengthened the survival times of the tumor-bearing nude mice (Supplemental Figure 27, A and B). Taken together, these results demonstrate that VEGF-C signaling blocking reverses BLACAT2-induced lymphangiogenesis and $\mathrm{LN}$ metastasis in vivo (Figure 10D).

\section{Discussion}

Our study represents what we believe is the first analysis of lncRNA-guided epigenetic regulation of VEGF-C and lymphatic 
A

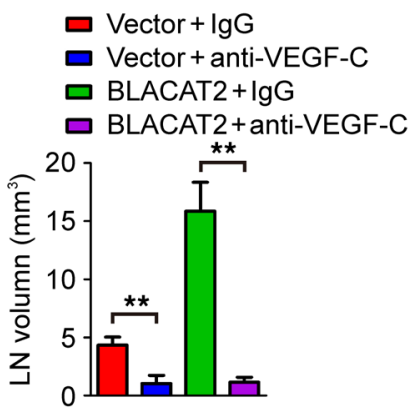

B

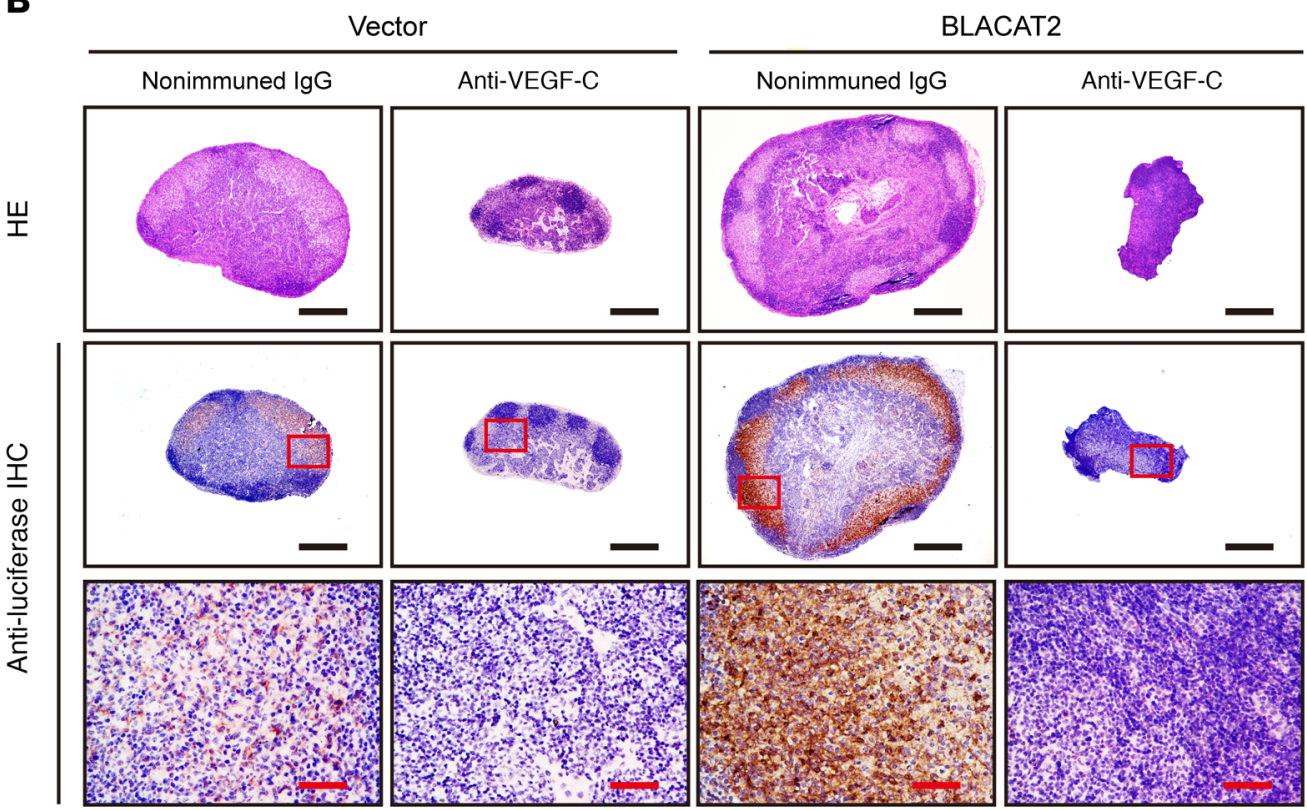

C

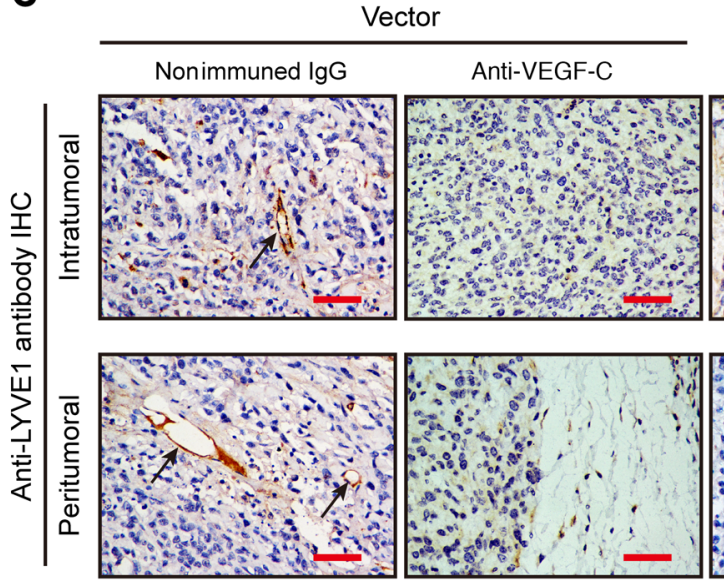

BLACAT2

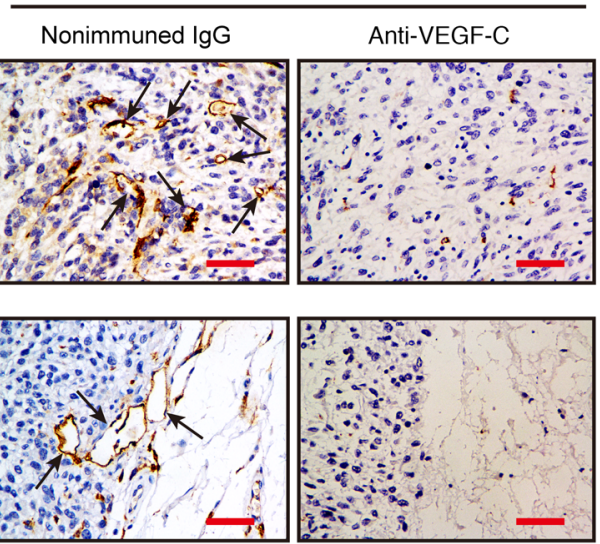

- Vector $+\operatorname{lgG}$

- Vector+anti-VEGF-C

口 BLACAT2 + IgG

ㅁ BLACAT2 + anti-VEGF-C

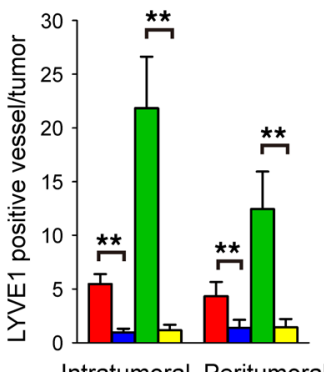

D
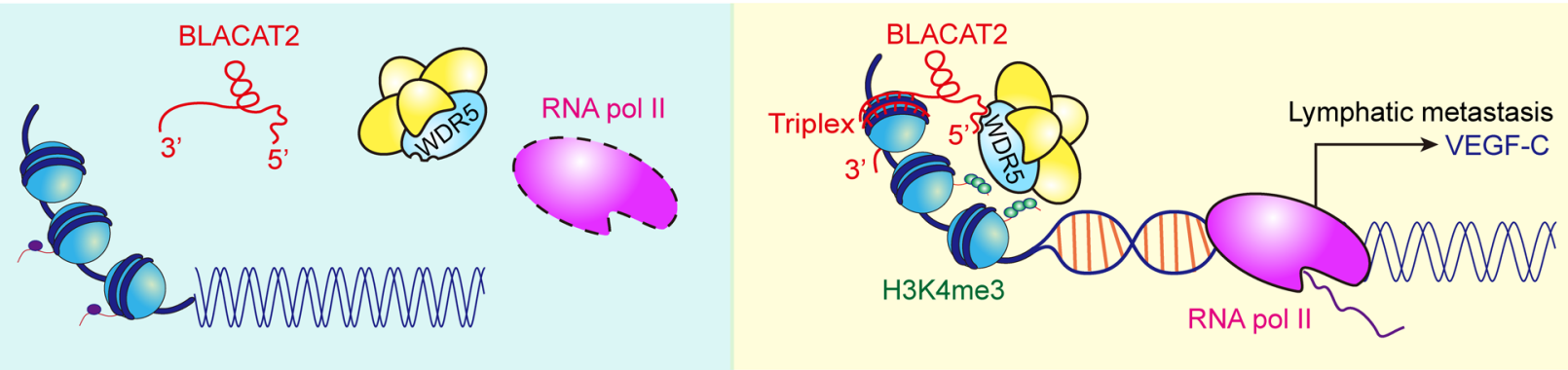

Figure 10. Inhibition of VEGF-C with neutralizing antibody abrogates BLACAT2-induced LN metastasis in vivo. (A) Volume quantification (right panel) of popliteal LN metastasis after inhibition of VEGF-C with neutralizing antibody. Popliteal LNs were enucleated and analyzed at the time of death or after 60 days ( $n=12$ per group). (B) Representative images of H\&E staining and IHC staining confirming LN status $(n=12)$. Scale bars: $500 \mu \mathrm{m}$ (black); $50 \mu \mathrm{m}$ (red). (C) Representative images (left panel) of IHC staining evaluating microlymphatic vessel density with anti-LYVE1 (left panels, as indicated with black arrows) and histogram analysis (right panel) of primary tumors from the footpads of nude mice. Scale bars: $50 \mu \mathrm{m}$. Error bars represent SD of the mean, ${ }^{* *} P<0.01$, Student's $t$ test (A and $\mathbf{C}$ ). (D) Proposed model of the role of BLACAT2 in LN metastasis of bladder cancer. 
metastasis in bladder cancer. We showed that BLACAT2, an lncRNA, was markedly upregulated in human LN-metastatic bladder cancer. BLACAT2 overexpression epigenetically induced intratumoral/peritumoral lymphangiogenesis and invasion of bladder cancer through a direct interaction with WDR5, a core component of the histone H3K4 methyltransferase complex. Inhibition of lymphangiogenesis using a VEGF-C antibody markedly suppressed LN metastasis of BLACAT2-overexpressing bladder cancer cells in vivo and prolonged the survival times of tumor-bearing mice. These results provide mechanistic and translational insights into the lymphatic metastasis of bladder cancer and suggest that the inhibition of lymphangiogenesis using a VEGF-C antibody may serve as a potential tailored treatment for patients with BLACAT2overexpressing bladder cancers.

Lymphangiogenesis, a common early metastatic event, is critical for LN metastasis and is the strongest prognostic marker of survival for patients with bladder cancer $(10,12)$. Cancer cells induce the formation of new lymphatic vessels, which are characterized by discontinuous cell-cell junctions and a lack of pericytes or vascular smooth muscle cells, via secretion of lymphangiogenic growth factors with promotion of tumor cell dissemination to LNs $(29,42)$. Importantly, diminishing lymphangiogenesis in vivo prevents regional LN metastasis and prolongs animal survival times in studies of other cancer types $(43,44)$. Herein, our results show that downregulation of BLACAT2 plays dual antilymphatic metastasis roles, including prevention of lymphangiogenesis and inhibition of cell invasiveness, suggesting that BLACAT2 may serve as a potential target for intervention in bladder cancer. To our knowledge, this is the first report to show that overexpression of an lncRNA closely correlates with lymphangiogenesis and LN metastasis in bladder cancer and predicts a poor prognosis of patients with MIBC.

Recently, small molecules that were designed to target the folded structure of the oncogenic noncoding RNA have shown significant antitumor effects in vivo by selectively modulating noncoding RNAs in cancer cells with no effects in normal cells (45). Additionally, locked nucleic acid (LNA) can serve as a therapeutic agent that specifically targets lncRNAs in vivo (46). In the future, inhibition of tumor-induced lymphangiogenesis and cancer invasiveness via small molecules that specifically target BLACAT2 therefore might be a potential therapeutic target of human cancer.

VEGF-C, a lymphatic vessel-specific growth factor, is upregulated in various human cancers, including bladder cancer (47-49), and has been shown to play critical roles in disrupting the endothelial lymphatic barrier $(50,51)$ and facilitating lymphatic invasion of cancer cells, ultimately resulting in significantly enhanced lymphatic metastasis and cancer treatment failure (52). Blocking the VEGF-C/VEGFR-3 lymphangiogenic axis significantly reduces the rate of LN metastasis in multiple cancer-bearing experimental mouse models $(17,44,53)$. Interestingly, several studies have suggested that the inhibition of VEGF-C not only decreases metastatic lesions within LNs in mouse models, but also suppresses metastatic cancer dissemination from LNs to distant organs $(9,15$, $41,54)$. Moreover, a VEGF-C monoclonal antibody (VGX-100) has been tested in a phase I clinical trial for the treatment of advanced or metastatic solid tumors (ClinicalTrials.gov NCT01514123). Therefore, exploring the precise molecular mechanism through which VEGF-C is overexpressed in bladder cancer would provide a potential predictor for effective anti-VEGF-C treatment. Herein, we have identified an IncRNA, BLACAT2, which contributes to lymphangiogenesis and lymphatic metastasis by epigenetically upregulating VEGF-C expression in bladder cancer. Our results demonstrate that BLACAT2 directly associates with the VEGF-C promoter via formation of triplexes. Moreover, depletion of VEGF-C using siRNA or a neutralizing antibody (pV1006R-r) showed promising antitumor effects in BLACAT2-overexpressing bladder cancer by inhibiting lymphangiogenesis and LN metastasis. Therefore, our findings uncover a molecular mechanism for the lymphatic metastasis of bladder cancer and suggest that BLACAT2 might serve as a potential biomarker for clinical intervention in LN-metastatic bladder cancer.

The role of BLACAT2 in mediating the interaction of WDR5 with multiple promoter target sequences of VEGF-C is an interesting paradigm. WDR5, a core component of the histone H3K4 methyltransferase complex, is crucial for vertebrate development and plays an important role in the self-renewal of pluripotent cells and cell differentiation $(39,55)$. Multiple studies have recently documented that dysregulated WDR5 is involved in promoting cancer metastasis and chemoresistance $(56,57)$, suggesting that WDR5 may serve as a therapeutic target for cancer (58). However, WDR5 is extensively expressed in numerous cell types and plays important roles in various biological functions $(39,55,59,60)$. Therefore, inhibition of WDR5 signaling may result in broad disturbances in normal cellular functions. Additionally, the WDR5 protein itself lacks putative DNA-binding domains, so the mechanisms through which WDR 5 orients itself to its target sites across the chromatin are largely unknown. Herein, we propose a model in which BLACAT2 directly binds to WDR5 protein and recruits it to specific genomic loci through the formation of a triplex with target DNA sequences. This finding may explain why chromatin-modifying complexes can bind to numerous gene promoters in a sequence-specific manner with limited binding domains. Interestingly, a previous study has shown that point mutations on the lncRNA-binding sites of WDR5 do not affect the intrinsic catalytic activity and mixed lineage leukemia (MLL) complex assembly, but significantly abolish the binding efficiency of WDR5 to chromatin (55), indicating that blocking the interaction between lncRNA and WDR 5 may produce more specific effects than targeting WDR5 itself.

In summary, based on our results, we propose a model in which the overexpression of an IncRNA, BLACAT2, promotes LN metastasis by inducing tumor-associated lymphangiogenesis and promoting bladder cancer cell invasiveness. Moreover, analyses of TCGA and GEO databases have shown that BLACAT2 is also elevated in multiple types of human cancer, suggesting the oncogenic role of BLACAT2. Therefore, it is worth delineating the epigenetic regulatory mechanisms of the BLACAT2/WDR5 complex in relation to downstream targets in other human cancer types.

\section{Methods}

Human tissue samples and study approval. Formalin-fixed, paraffinembedded (FFPE) tissues and snap-frozen fresh bladder cancer tissues and normal adjacent tissues were obtained from patients who underwent surgery at Sun Yat-sen Memorial Hospital. All samples were confirmed by 2 pathologists. 
Microarray and data analysis, cell culture and transfection, RNA isolation, RT-qPCR analysis, nuclear fractionation, cell motility, invasion assay, and immunoblots. Microarray and data analyses, cell culture and transfections, RNA isolations, RT-qPCR analysis, nuclear fractionations, cell motility and invasion assays, and immunoblots were performed following standard protocols as previously reported (20). All details are also described in the Supplemental Materials and Methods section. The SurePrint G3 Human Gene Expression Microarray (catalog G4851B, Agilent) was used for the microarray analysis. Patient characteristics are described in Supplemental Table 1. All bladder cancer cell lines were purchased from ATCC. The sequences of siRNA oligos are listed in Supplemental Table 4. The sequences of all primers used in this study are shown in Supplemental Table 5. For the overall and metastasis-free survival analyses, the median expression level of BLACAT2 determined by RT-qPCR was used as a cutoff value.

ISH and immunohistochemistry analysis. BLACAT2 expression was also examined using ISH in FFPE samples, as previously described (61). Briefly, after dewaxing and rehydration, the samples were digested with $20 \mu \mathrm{g} / \mathrm{ml}$ proteinase $\mathrm{K}$ (Roche), fixed in $4 \%$ paraformaldehyde, hybridized with a double ( $5^{\prime}$ and $3^{\prime}$ ) digoxin-labeled (DIGlabeled) LNA-modified BLACAT2 probe (Exiqon) at $42^{\circ} \mathrm{C}$ overnight, and subsequently incubated overnight at $4^{\circ} \mathrm{C}$ with anti-DIG monoclonal antibody conjugated to alkaline phosphatase (Roche, catalog 11093274910). After staining with nitro blue tetrazolium/5-bromo4-chloro-3-indolylphosphate (Roche), the sections were mounted and observed. A double (5' and $3^{\prime}$ ) DIG-labeled scrambled probe was used as the negative control, and double ( $5^{\prime}$ and $3^{\prime}$ ) DIG-labeled probes against U6 snRNA (nuclear staining) and $\beta$-actin (cytoplasmic staining) were used as technical positive controls. The sequences of all the probes used in ISH are shown in Supplemental Table 6.

The immunohistochemistry analyses were performed as previously described (62). The degree of ISH and immunohistochemistry (IHC) in the FFPE samples was reviewed and scored by 2 independent pathologists. The proportion of tumor cells with positive staining was designated as follows: 0 , no positive tumor cells; $1,0 \%-10 \%$ positive tumor cells; 2 , $10 \%-30 \%$ positive tumor cells; $3,30 \%-70 \%$ positive tumor cells; and 4 , more than $70 \%$ positive tumor cells. The staining intensity was graded as follows: 1, no staining; 2, weak staining (light yellow for IHC, light blue for ISH); 3, moderate staining (yellow brown for IHC, moderate blue for ISH); and 4, strong staining (brown for IHC, strong blue for ISH). The staining index (SI) was calculated by multiplying the proportion of positive tumor cells and the staining intensity score, with possible scores of $\mathrm{O}$, $1,2,3,4,6,8,9,12$, and 16 . ISH and IHC were independently analyzed by 2 experienced pathologists who were blinded to the tissue information.

Popliteal LN metastasis assay. BALB/c nude mice (4 to 5 weeks old) were purchased from the Experimental Animal Center, Sun Yat-sen University. Eight mice were included in each group, and lentivirustransduced UM-UC- 3 cells $\left(5 \times 10^{5}\right.$ cells $)$ that stably expressed firefly luciferase were inoculated into the footpads. For the VEGF-C-treatment assays, a nonimmune control $\operatorname{IgG}(50 \mathrm{ng} / \mathrm{ml}$ in HBSS) or neutralizing antibody against VEGF-C (pV1006R-r, $50 \mathrm{ng} / \mathrm{ml}$ in HBSS) was intravenously injected. The primary tumors and popliteal LNs were enucleated and embedded in paraffin. The $\mathrm{LN}$ volumes were calculated using the following formula: $\mathrm{LN}$ volume $\left(\mathrm{mm}^{3}\right)=($ length $[\mathrm{mm}]) \times(\text { width }[\mathrm{mm}])^{2}$ $\times$ 0.52. The FFPE samples were analyzed by IHC with anti-LYVE-1 and anti-luciferase antibodies (Abcam). Images were captured using a Nikon Eclipse 80i system with NIS-Elements software (Nikon).
RIP and ChIP. RIP assays were performed using the EZ-Magna RIP Kit (Millipore) and ChIP experiments were performed using the EZ-Magna ChIP A/G Kit (Millipore) at $4^{\circ} \mathrm{C}$ as previously described (20). Briefly, for the RIP assays, $1 \times 10^{7}$ cells were harvested and lysed with RIP lysis buffer with 1 freeze-thaw cycle. Cell extracts were coimmunoprecipitated using anti-WDR5 (Abcam, ChIP grade) and the retrieved RNA was quantified by RT-qPCR. Normal mouse IgG was used as the negative control. For RT-qPCR analysis, HOTTIP was used as the positive control and U1 RNA was used as a nonspecific control. For each ChIP assay, $1 \times 10^{6}$ cells were fixed in $1 \%$ formaldehyde at room temperature for 10 minutes and the nucleus was isolated with nuclear lysis buffer (Millipore) supplemented with a protease inhibitor cocktail (Millipore). Chromatin DNA was sonicated and sheared to a length between 200 bp and 1,000 bp. The sheared chromatin was immunoprecipitated at $4^{\circ} \mathrm{C}$ overnight using anti-WDR5 (Abcam, ChIP grade) or anti-H3K4me3 (Abcam, ChIP grade). Normal mouse IgG was used as the negative control, and anti-RNA pol II (Millipore) was used as a technical control antibody. Primers for ChIP-qPCR are listed in Supplemental Table 5.

Chromatin isolation by RNA purification. ChIRP was performed as previously described (63). Briefly, each probe was designed using a single-molecule FISH online designer (63), biotin-labeled at its $3^{\prime}$ terminus, and divided into odd or even groups. Next, $2 \times 10^{7}$ cells were used for each ChIRP assay and crosslinked in $1 \%$ formaldehyde at room temperature for 10 minutes. The cells were lysed, sheared to 100-500 bp fragments with mean sizes of approximately 150-200 bp at $4^{\circ} \mathrm{C}$, and hybridized with probes in a hybridization oven. The probes used in the ChIRP assay are listed in Supplemental Table 6.

Accession numbers. The accession numbers for the full-length human BLACAT2 sequence were submitted to GenBank (MF435912, MF435913, MF435914). All primary data from microarray analysis and NGS data were deposited in the NCBI's Gene Expression Omnibus database (GSE100926, GSE101287).

Statistics. Quantitative data are presented as mean \pm SD from at least 3 independent experiments. Unless otherwise noted, differences between 2 groups were analyzed with the unpaired/paired Student's $t$ test using SPSS 13.0. The Mann-Whitney $U$ test was used for independent samples when the population could not be assumed to be normally distributed. When more than 2 treatments were compared, 1-way ANOVA followed by Dunnett's multiple comparisons tests was performed. The correlation coefficient between BLACAT2 expression and clinicopathological characteristics was calculated using the SPSS 13.0 Spearman's and Kendall's correlation. Kaplan-Meier curves of metastasis-free survival or overall survival were compared with the log-rank test. All tests were 2 sided. Differences were considered statistically significant at $P<0.05$.

Study approval. Ethical consent was given by the Sun Yat-sen University Committee for Ethical Review of Research Involving Human Subjects. The use of human bladder cancer tissue specimens was evaluated and approved by the Ethical Committee of Sun Yat-sen Memorial Hospital, and written informed consent was obtained from all participants or their appropriate surrogates. All animal studies were conducted with the approval of the Sun Yat-sen University Institutional Animal Care and Use Committee and were performed in accordance with established guidelines.

\section{Author contributions}

$\mathrm{WH}, \mathrm{JH}$ and TL participated in the study design; GZ, NJ and CC carried out the in vitro and in vivo experiments and data anal- 
ysis; XF and XC performed the clinical data analysis; and $\mathrm{WH}$ wrote the manuscript. All the authors read and approved the final manuscript.

\section{Acknowledgments}

This study was funded by the National Natural Science Foundation of China (Grant No. 81572514, 81472384, 81472381, 81402106, 81772719, 81772728, 91740119); Guangdong Science and Technology Development Fund (2017B020227007); Science and Technology Program of Guangzhou (Grant No. 201604020156, 201604020177, 201707010116); National Natural Science Foundation of Guangdong (Grant No. 2016A030313321, 2015A030311011, 2015A030310122, 2014A030310133, S2013020012671, 07117336, 10151008901000024), "Three Big Constructions" funds of Sun Yat-sen University; Project Supported by Guangdong Province Higher Vocational Colleges \&
Schools Pearl River Scholar Funded Scheme; Elite Young Scholars Program of Sun Yat-Sen Memorial Hospital (J201401, J2015106); Fundamental Research Funds for Young Teachers in the Higher Education Institutions of China (16ykpy12); National Clinical Key Specialty Construction Project for Department of Urology and Department of Oncology. The Key Laboratory of Malignant Tumor Gene Regulation and Target Therapy of Guangdong Higher Education Institutes, Sun-Yat-Sen University (Grant KLB09001); Key Laboratory of Malignant Tumor Molecular Mechanism and Translational Medicine of Guangzhou Bureau of Science and Information Technology ([2013]163).

Address correspondence to: Tianxin Lin, Department of Urology, Sun Yat-sen Memorial Hospital, 107th Yanjiangxi Road, Yuexiu District, Guangzhou, Guangdong Province, China, postal code: 510120. Phone: 86.20.34070447; Email: lintx@mail.sysu.edu.cn.
1. Torre LA, Bray F, Siegel RL, Ferlay J, LortetTieulent J, Jemal A. Global cancer statistics, 2012. CA Cancer J Clin. 2015;65(2):87-108.

2. Wu XR. Urothelial tumorigenesis: a tale of divergent pathways. Nat Rev Cancer. 2005;5(9):713-725.

3. Hautmann RE, de Petriconi RC, Pfeiffer C, Volkmer BG. Radical cystectomy for urothelial carcinoma of the bladder without neoadjuvant or adjuvant therapy: long-term results in 1100 patients. Eur Urol. 2012;61(5):1039-1047.

4. Fajkovic H, et al. Extranodal extension is a powerful prognostic factor in bladder cancer patients with lymph node metastasis. Eur Urol. 2013;64(5):837-845.

5. Zargar-Shoshtari K, et al. A multi-institutional analysis of outcomes of patients with clinically node positive urothelial bladder cancer treated with induction chemotherapy and radical cystectomy. JUrol. 2016;195(1):53-59.

6. Raza SJ, et al. Oncologic outcomes following robot-assisted radical cystectomy with minimum 5-year follow-up: the Roswell Park cancer institute experience. Eur Urol. 2014;66(5):920-928.

7. Cao Y. Opinion: emerging mechanisms of tumour lymphangiogenesis and lymphatic metastasis. Nat Rev Cancer. 2005;5(9):735-743.

8. Karaman S, Detmar M. Mechanisms of lymphatic metastasis. JClin Invest. 2014;124(3):922-928.

9. Mandriota SJ, et al. Vascular endothelial growth factor-C-mediated lymphangiogenesis promotes tumour metastasis. EMBO J. 2001;20(4):672-682.

10. Fernández MI, et al. Prognostic implications of lymphangiogenesis in muscle-invasive transitional cell carcinoma of the bladder. Eur Urol. 2008;53(3):571-578.

11. Zhou M, He L, Zu X, Zhang H, Zeng H, Qi L. Lymphatic vessel density as a predictor of lymph node metastasis and its relationship with prognosis in urothelial carcinoma of the bladder. BJU Int. 2011;107(12):1930-1935.

12. Miyata $Y$, et al. Lymphangiogenesis and angiogenesis in bladder cancer: prognostic implications and regulation by vascular endothelial growth factors-A, -C, and -D. Clin Cancer Res. 2006;12(3 Pt 1):800-806.
13. Keck B, et al. Neuropilin-2 and its ligand VEGF-C predict treatment response after transurethral resection and radiochemotherapy in bladder cancer patients. Int J Cancer. 2015;136(2):443-451.

14. Chen JC, Chang YW, Hong CC, Yu YH, Su JL. The role of the VEGF-C/VEGFRs axis in tumor progression and therapy. Int JMol Sci. 2012;14(1):88-107.

15. Karpanen T, et al. Vascular endothelial growth factor $\mathrm{C}$ promotes tumor lymphangiogenesis and intralymphatic tumor growth. Cancer Res. 2001;61(5):1786-1790.

16. Rinderknecht M, Villa A, Ballmer-Hofer K, Neri D, Detmar M. Phage-derived fully human monoclonal antibody fragments to human vascular endothelial growth factor-C block its interaction with VEGF receptor-2 and 3. PLoS One. 2010;5(8):e11941.

17. Chen $\mathrm{Y}$, et al. Vascular endothelial growth factor$\mathrm{C}$ promotes the growth and invasion of gallbladder cancer via an autocrine mechanism. Mol Cell Biochem. 2010;345(1-2):77-89.

18. He Y, et al. Suppression of tumor lymphangiogenesis and lymph node metastasis by blocking vascular endothelial growth factor receptor $3 \mathrm{sig}$ naling. J Natl Cancer Inst. 2002;94(11):819-825.

19. Evans JR, Feng FY, Chinnaiyan AM. The bright side of dark matter: lncRNAs in cancer. J Clin Invest. 2016;126(8):2775-2782.

20. He W, et al. linc-UBC1 physically associates with polycomb repressive complex 2 (PRC2) and acts as a negative prognostic factor for lymph node metastasis and survival in bladder cancer. Biochim Biophys Acta. 2013;1832(10):1528-1537.

21. Prensner JR, et al. The long noncoding RNA SChLAP1 promotes aggressive prostate cancer and antagonizes the SWI/SNF complex. Nat Genet. 2013;45(11):1392-1398.

22. Gupta RA, et al. Long non-coding RNA HOTAIR reprograms chromatin state to promote cancer metastasis. Nature. 2010;464(7291):1071-1076.

23. Hao Y, Yang X, Zhang D, Luo J, Chen R. Long noncoding RNA LINC01186, regulated by TGF- $\beta$ /SMAD3, inhibits migration and invasion through epithelial-mesenchymal-transition in lung cancer. Gene. 2017;608:1-12.

24. Yuan JH, et al. A long noncoding RNA activated by TGF- $\beta$ promotes the invasion-metastasis cascade in hepatocellular carcinoma. Cancer Cell. 2014;25(5):666-681.

25. Shang C, et al. Silence of cancer susceptibility candidate 9 inhibits gastric cancer and reverses chemoresistance. Oncotarget. 2017;8(9):15393-15398.

26. Crea F, et al. The role of epigenetics and long noncoding RNA MIAT in neuroendocrine prostate cancer. Epigenomics. 2016;8(5):721-731.

27. Zhou C, et al. LncRNA MEG3 downregulation mediated by DNMT3b contributes to nickel malignant transformation of human bronchial epithelial cells via modulating PHLPP1 transcription and HIF-1 $\alpha$ translation. Oncogene. 2017;36(27):3878-3889.

28. Kong J, et al. Long non-coding RNA LINC01133 inhibits epithelial-mesenchymal transition and metastasis in colorectal cancer by interacting with SRSF6. Cancer Lett. 2016;380(2):476-484.

29. Karaman S, Detmar M. Mechanisms of lymphatic metastasis. JClin Invest. 2014;124(3):922-928.

30. Stacker SA, Williams SP, Karnezis T, Shayan R, Fox SB, Achen MG. Lymphangiogenesis and lymphatic vessel remodelling in cancer. Nat Rev Cancer. 2014;14(3):159-172.

31. Atmaca A, et al. SNAI2/SLUG and estrogen receptor mRNA expression are inversely correlated and prognostic of patient outcome in metastatic non-small cell lung cancer. BMC Cancer. 2015;15:300.

32. Šelemetjev S, Đoric I, Paunovic I, Tatic S, Cvejic D. Coexpressed high levels of VEGF-C and active MMP-9 are associated with lymphatic spreading and local invasiveness of papillary thyroid carcinoma. Am J Clin Pathol. 2016;146(5):594-602.

33. Szarvas T, vom Dorp F, Ergün S, Rübben H. Matrix metalloproteinases and their clinical relevance in urinary bladder cancer. Nat Rev Urol. 2011;8(5):241-254.

34. Suzuki K, Morita T, Tokue A. Vascular endothelia growth factor-C (VEGF-C) expression predicts lymph node metastasis of transitional cell carcinoma of the bladder. Int J Urol. 2005;12(2):152-158.

35. Côme C, et al. Snail and slug play distinct roles during breast carcinoma progression. Clin Cancer Res. 2006;12(18):5395-5402. 
36. He S, Zhang H, Liu H, Zhu H. LongTarget: a tool to predict lncRNA DNA-binding motifs and binding sites via Hoogsteen base-pairing analysis. Bioinformatics. 2015;31(2):178-186.

37. Grote P, Herrmann BG. The long non-coding RNA Fendrr links epigenetic control mechanisms to gene regulatory networks in mammalian embryogenesis. RNA Biol. 2013;10(10):1579-1585.

38. Schuettengruber B, Chourrout D, Vervoort M, Leblanc B, Cavalli G. Genome regulation by polycomb and trithorax proteins. Cell. 2007;128(4):735-745.

39. Wysocka J, et al. WDR5 associates with histone $\mathrm{H} 3$ methylated at $\mathrm{K} 4$ and is essential for H3 K4 methylation and vertebrate development. Cell. 2005;121(6):859-872.

40. Wang CA, et al. SIX1 induces lymphangiogenesis and metastasis via upregulation of VEGF-C in mouse models of breast cancer. J Clin Invest. 2012;122(5):1895-1906.

41. Shibata MA, Shibata E, Morimoto J, HaradaShiba M. Therapy with siRNA for Vegf-c but not for Vegf-d suppresses wide-spectrum organ metastasis in an immunocompetent xenograft model of metastatic mammary cancer. Anticancer Res. 2013;33(10):4237-4247.

42. Zheng W, Aspelund A, Alitalo K. Lymphangiogenic factors, mechanisms, and applications. J Clin Invest. 2014;124(3):878-887.

43. Patel V, et al. Decreased lymphangiogenesis and lymph node metastasis by mTOR inhibition in head and neck cancer. Cancer Res. 2011;71(22):7103-7112.

44. Pytowski B, et al. Complete and specific inhibition of adult lymphatic regeneration by a novel
VEGFR-3 neutralizing antibody. J Natl Cancer Inst. 2005;97(1):14-21.

45. Velagapudi SP, et al. Design of a small molecule against an oncogenic noncoding RNA. Proc Natl Acad Sci U S A. 2016;113(21):5898-5903.

46. Li C, et al. A ROR1-HER3-lncRNA signalling axis modulates the Hippo-YAP pathway to regulate bone metastasis. Nat Cell Biol. 2017;19(2):106-119.

47. Cao Y, Zhong W. Tumor-derived lymphangiogenic factors and lymphatic metastasis. Biomed Pharmacother. 2007;61(9):534-539.

48. Yonemura Y, et al. Role of vascular endothelial growth factor $\mathrm{C}$ expression in the development of lymph node metastasis in gastric cancer. Clin Cancer Res. 1999;5(7):1823-1829.

49. Salven $P$, et al. Vascular endothelial growth factors VEGF-B and VEGF-C are expressed in human tumors. Am J Pathol. 1998;153(1):103-108.

50. Tacconi C, et al. Vascular endothelial growth factor $\mathrm{C}$ disrupts the endothelial lymphatic barrier to promote colorectal cancer invasion. Gastroenterology. 2015;148(7):1438-51.e8.

51. Breslin JW, Yuan SY, Wu MH. VEGF-C alters barrier function of cultured lymphatic endothelial cells through a VEGFR-3-dependent mechanism. Lymphat Res Biol. 2007;5(2):105-113.

52. Dufies M, et al. Sunitinib stimulates expression of VEGFC by tumor cells and promotes lymphangiogenesis in clear cell renal cell carcinomas. Cancer Res. 2017;77(5):1212-1226.

53. Burton JB, et al. Suppression of prostate cancer nodal and systemic metastasis by blockade of the lymphangiogenic axis. Cancer Res. 2008;68(19):7828-7837.

54. Gogineni A, et al. Inhibition of VEGF-C modu- lates distal lymphatic remodeling and secondary metastasis. PLoS ONE. 2013;8(7):e68755.

55. Yang YW, et al. Essential role of lncRNA binding for WDR5 maintenance of active chromatin and embryonic stem cell pluripotency. Elife. 2014;3:e02046.

56. Wu MZ, et al. Interplay between HDAC3 and WDR5 is essential for hypoxia-induced epithelial-mesenchymal transition. Mol Cell. 2011;43(5):811-822.

57. Chen X, et al. Upregulated WDR5 promotes proliferation, self-renewal and chemoresistance in bladder cancer via mediating H3K4 trimethylation. Sci Rep. 2015;5:8293.

58. Grebien F, et al. Pharmacological targeting of the Wdr5-MLL interaction in C/EBP $\alpha \mathrm{N}$-terminal leukemia. Nat Chem Biol. 2015;11(8):571-578.

59. Zhu ED, Demay MB, Gori F. Wdr5 is essential for osteoblast differentiation. JBiol Chem. 2008;283(12):7361-7367.

60. Ang YS, et al. Wdr5 mediates self-renewal and reprogramming via the embryonic stem cell core transcriptional network. Cell. 2011;145(2):183-197.

61. Nie Y, Liu X, Qu S, Song E, Zou H, Gong C. Long non-coding RNA HOTAIR is an independent prognostic marker for nasopharyngeal carcinoma progression and survival. Cancer Sci. 2013;104(4):458-464.

62. Liu L, et al. TBL1XR1 promotes lymphangiogenesis and lymphatic metastasis in esophageal squamous cell carcinoma. Gut. 2015;64(1):26-36.

63. Chu C, Chang HY. Understanding RNAchromatin interactions using chromatin isolation by RNA purification (ChIRP). Methods Mol Biol. 2016;1480:115-123. 